\title{
MINIMALITY IN DIAGRAMS OF SIMPLICIAL SETS
}

\author{
CARLES BROTO, RAMÓN FLORES, AND CARLOS GIRALDO
}

\begin{abstract}
We formulate the concept of minimal fibration in the context of fibrations in the model category $\mathbf{S}^{\mathcal{C}}$ of $\mathcal{C}$-diagrams of simplicial sets, for a small index category $\mathcal{C}$. When $\mathcal{C}$ is an $E I$-category satisfying some mild finiteness restrictions, we show that every fibration of $\mathcal{C}$-diagrams admits a well-behaved minimal model. As a consequence, we establish a classification theorem for fibrations in $\mathbf{S}^{\mathcal{C}}$ over a constant diagram, generalizing the classification theorem of Barratt, Gugenheim, and Moore for simplicial fibrations [1].
\end{abstract}

\section{IntroduCtion}

After the introduction by D. Kan in the fifties of the theory of simplicial sets and Kan fibrations, M.G. Barratt, V.K. Gugenheim, and J.C.Moore developed in their celebrated paper [1] the appropriate version of simplicial fibre bundles, which in particular are Kan fibrations when the fibre is a Kan complex. Conversely, given a Kan fibration, these authors proposed the notion of a minimal simplicial fibration, a fibrewise deformation retract of the original fibre map, which always exists and is itself a simplicial fibre bundle, provided the base of the fibration is connected. In this way, the classification of simplicial fibrations reduces to the classification of simplicial fibre bundles. The latter is achieved by associating to every fibre bundle a twisted cartesian product (TCP), where the twisting functions take values in the structure group $G$ of the bundle. Then, the simplicial group $G$ provides a classifying space $\bar{W}(G)$ and a universal fibre bundle such that every $G$-bundle over a base $B$ can be obtained as a pullback of a map $B \rightarrow \bar{W}(G)$ along this universal bundle.

Denote by $\mathbf{S}$ the category of simplicial sets. Given a small category $\mathcal{C}$, the main goal of our work has been to extend Barratt-Gugenheim-Moore framework to the category $\mathbf{S}^{\mathcal{C}}$ of $\mathcal{C}$-diagrams of simplicial sets assuming that the fibrations involved are defined over a constant base. The first observation is that the category of diagrams possess a structure of cofibrantly generated model category and inherits a simplicial structure from $\mathbf{S}$ [12]. Then, assuming always a constant base $B$, there exist natural translations of the notions of simplicial fibration, simplicial bundle or twisted cartesian product.

Extending the notion of minimal fibration is more involved. To this aim we define a full subcategory $\Gamma$ of $\mathbf{S}^{\mathcal{C}}$ whose set of objects is given by the free $\mathcal{C}$-diagrams $\delta_{n}^{c}$ over the standard $n$-simplex $\Delta[n]$ (see Definition 2.2). Identifying $\mathcal{C}$-diagrams with $\Gamma^{\mathrm{op}}$-sets, the free diagrams play in this context the role of the standard simplices in the classical theory, and become the basic building blocks throughout we may study any $\mathcal{C}$-diagram $X$. In particular, a $n$-csimplex will simply be a natural transformation from a free diagram $\delta_{n}^{c}$ to $X$, for any $n \geq 0$ and $c \in \mathrm{Ob}(\mathcal{C})$,

Now it is possible to define a sub-p-homotopy relation for the set of $n$-c-simplices of the total space of fibration of $\mathcal{C}$-diagrams for all $n \geq 0$ and $c \in \mathcal{C}$ (Definition 3.2), and then the notion of minimal fibration (Definition 3.3). If $\mathcal{C}$ is an arbitrary small category the shape

C. Broto and C. Giraldo are partially supported by FEDER-MINECO Grant MTM2016-80439-P and AGAUR Grant 2017SGR1725.

R. Flores is partially supported by FEDER-MINECO Grant MTM2016-76453-C2-1-P. 
of the orbits of a free diagram can be elusive. However, when $\mathcal{C}$ is an artinian EI-category (Definition 3.5), our main theorem establishes the existence of minimal $\mathcal{C}$-fibrations:

Theorem A. Let $\mathcal{C}$ be a small artinian EI-category. If $p: X \longrightarrow B$ is a fibration in $\mathbf{S}^{\mathcal{C}}$ for which $X$ is a free diagram, then $p$ has a strong fibrewise deformation retract, $q: \hat{X} \longrightarrow B$, which is minimal and where $\hat{X}$ is a free $\mathcal{C}$-diagram.

Observe that Example 3.13 shows that some restrictions on the structure of the index category are unavoidable in order to always find minimal models for the fibrations.

Now consider a simplicial set $B$, a category $\mathcal{C}$, and the composition $\mathcal{C} \rightarrow\{*\} \rightarrow \mathbf{S}$ of the trivial functor with the functor that takes the point to $B$. Slightly abusing notation, we will also denote this diagram by $B$ and call it a $\mathcal{C}$-diagram over a constant base $B$. Now, Theorem $\mathrm{A}$ opens the way to extend all the Barrat-Gugenheim-Moore program to $\mathcal{C}$-diagrams over a constant base. As an intermediate step, we prove that that any $\mathcal{C}$-fibre bundle over a constant diagram $B$ with fibre $F$ is equivalent to a $\mathcal{C}$-twisted cartesian product (and viceversa), and this allows to classify $\mathcal{C}$-fibre bundles. We denote by $\operatorname{aut}_{\mathcal{C}}(F)$ the simplicial group of automorphisms of the diagram $F$.

Theorem B. Let $\mathcal{C}$ be a small category, $F$ a $\mathcal{C}$-diagram, $G$ a simplicial subgroup of aut $t_{\mathcal{C}}(F)$ and $B$ a simplicial set. Then the homotopy classes of maps $[B, \bar{W} G]$ from $B$ to $\bar{W} G$ are in bijective correspondence with the equivalence classes of $\mathcal{C}$-fibre bundles with fibre $F$, constant base $B$ and group $G$.

Now we can use Theorem $\underline{\mathrm{A}}$, Theorem $\mathrm{B}$, and Quillen's small object argument to classify fibrations in $\mathbf{S}^{\mathcal{C}}$.

Theorem C. Let $\mathcal{C}$ be a small artinian EI-category, $B$ a connected simplicial set, and $F$ a $\mathcal{C}$-diagram. Then there is a bijective correspondence between the set $\left[B, B\right.$ haut $\left._{\mathcal{C}}(F)\right]$ of homotopy classes of maps from $B$ to $B$ haut $_{\mathcal{C}}(F)$ and the set of weak homotopy classes of fibrations over the constant diagram $B$ whose fibres are weakly homotopy equivalent to $F$.

Roughly speaking, for a fibrant $\mathcal{C}$-diagram $F$, $\operatorname{haut}_{\mathcal{C}}(F)$ is $\operatorname{described}$ as the set of self equivalences of $F$ in the homotopy category that admit a rigidification in the category of simplicial sets. When $F$ is not fibrant we use a fibrant replacement. The precise definition appears in Section 5, and we also show there, elaborating on results of Dwyer-Kan [6], that it is a loop space.

This classification is related with the results of Dwyer-Kan-Smith in [7, classifying towers of fibrations. They construct inductively a classifying space $B\left(E Y_{1} \ldots E Y_{n}\right)$ by means of an appropriate homotopical version of the wreath product of groups. A tower of fibrations $X_{n} \longrightarrow X_{n-1} \longrightarrow \ldots \rightarrow X_{1} \longrightarrow X_{0}$, where $Y_{i}$ is the fibre of the map $X_{i} \longrightarrow X_{i-1}$, might be seen as a $\mathcal{C}$-fibration over the constant base $X_{0}$, where $\mathcal{C}$ is the finite poset $\{n \longrightarrow \ldots \longrightarrow 1\}$, and with fibre the diagram $F=F_{n} \longrightarrow F_{n-1} \longrightarrow \ldots \longrightarrow F_{1}$, being $F_{i}$ the fibre of the composite fibration $X_{i} \longrightarrow X_{0}$. With this interpretation, the tower is classified by $B$ haut $_{\mathcal{C}}(F)$, and this turns out to be a connected component of Dwyer-Kan-Smith classifying space.

Part of the motivation for our study arose from our attempts to dualize to the augmented case classical results ([8], [3]) of preservation of fibrations under localization functors. In this context, giving a fibration $F \rightarrow E \rightarrow B$ and a functor $L$, the problem is to produce another fibration $L F \rightarrow E^{\prime} \rightarrow B$ that is naturally mapped from the original one. Our research about this subject, which corresponds to the case of a diagram of two objects and one morphism, will appear in a separate paper. Observe that the base of the fibration does not change throughout the process. 
It is also worth mentioning the recent work of Blomgren-Chachólski [2]. Given objects $X$ and $F$, they define $\operatorname{Fib}(X, F)$ as the category whose objects are all the possible fibrations with base $X$ and fibre weakly homotopy equivalent to $F$. One of the innovative ideas of their work lies in the description of the homotopy type of $\operatorname{Fib}(X, F)$ and not only of its connected components, thus providing a refinement of the classical classification theorems. They develop the notion of core of a category in order to overcome set-theoretic difficulties that derive from the fact that $\operatorname{Fib}(X, F)$ is not a small category in general. Although their results are valid in any model category, the point of view is different from ours, as we propose a more classical and combinatorial approach in the model category of $\mathcal{C}$-diagrams.

The paper is structured as follows. In Section 2 we describe the structure of the category of diagrams of simplicial sets over a small index category. The theory of minimal fibrations in this category is developed in Section 3, where we prove Theorem A. Section 4 is devoted to discuss and classify $\mathcal{C}$-fibre bundles, a goal that is achieved in Theorem $\mathrm{B}$. Last section culminates the paper, by proving the classification result (Theorem C) for fibrations in the category of $\mathcal{C}$-diagrams. We conclude with an appendix, where we prove some technical properties of preordered sets that are needed in Section 3 .

Acknowledgments. The authors wish to thank Wojciech Chachólski for useful discussions about the paper. They also thank the Institute of Mathematics of the University of Seville and the Department of Mathematics of the Universitat Autònoma de Barcelona for their kind hospitality hosting joint meetings of them.

\section{The MOdel CATEGORY OF $\mathcal{C}$-DiAgrams}

The model category of $\mathcal{C}$-diagrams has been extensively investigated in [12]. In this section, we will first recall some basic facts about simplicial sets and will fix some notation (a more complete account of the theory can be found in [5, 10, 15]). Then, we explain how the category of $\mathcal{C}$-diagrams inherits the structure of cofibrantly generated simplicial model category from that of simplicial sets. In the way, we describe $\mathcal{C}$-diagrams as $\Gamma_{\mathcal{C}}^{\text {op }}$-sets (see Definition 2.2 below), a point of view that will be useful in the construction of minimal fibrations in Section 3 .

Let $\Delta$ be the category whose objects are the finite, totally ordered, non-empty sets $[n]=$ $\{0,1, \ldots, n\}, n \geq 0$, and whose morphisms are the order-preserving functions. A simplicial set $X$ is a functor $\Delta^{\mathrm{op}} \longrightarrow$ Sets, while a simplicial map is a natural transformation. We will denote by $\mathbf{S}$ the category of simplicial sets and simplicial maps.

A simplicial set can be seen as a sequence of sets $X=\left\{X_{n}\right\}_{n \geq 0}$, together with structural maps that consist of face operators $d_{i}: X_{n} \longrightarrow X_{n-1}, n \geq 1,0 \leq i \leq n$, and of degeneracy operators $s_{i}: X_{n} \longrightarrow X_{n+1}, n \geq 0,0 \leq i \leq n$, subject to the following relations:

$$
\begin{aligned}
d_{i} d_{j} & =d_{j-1} d_{i}, & & i<j \\
d_{i} s_{j} & =s_{j-1} d_{i}, & & i<j \\
d_{j} s_{j} & =1=d_{j+1} s_{j} & & \\
d_{i} s_{j} & =s_{j} d_{i-1}, & & i>j+1 \\
s_{i} s_{j} & =s_{j+1} s_{i}, & & i \leq j .
\end{aligned}
$$

Accordingly, a simplicial map $f: X \rightarrow Y$ between simplicial sets is a sequence of maps $f_{n}: X_{n} \longrightarrow Y_{n}$, for $n \geq 0$ which commutes with face and degeneracy operators. The set of simplicial maps from $X$ to $Y$ will be denoted by $\operatorname{Mor}_{\mathbf{S}}(X, Y)$. When no confusion is possible, a map between simplicial sets is understood to be a simplicial map. 
The elements of $X_{n}$ are called $n$-simplices, or just simplices if we do not need to emphasize the dimension $n$. Simplices in the image of a degeneracy operator are called degenerate simplices. In turn, all the simplices of the form $d_{i_{1}} d_{i_{2}} \ldots d_{i_{r}}(x), r \geq 0$ will be called faces of $x$. Those of the form $s_{i_{1}} s_{i_{2}} \ldots s_{i_{r}}(x), r \geq 1$, are called degeneracies of $x$.

The standard $n$-simplex $\Delta[n]$ is the simplicial set defined $\Delta[n]=\operatorname{Hom}_{\Delta}([-],[n])$. It contains a fundamental simplex $\imath_{n}=\operatorname{Id}_{[n]} \in \Delta[n]_{n}$ and all of simplices are either faces or degeneracies of $\imath_{n}$. Actually, Yoneda's lemma provides a natural isomorphism $\operatorname{Mor}_{\mathbf{S}}(\Delta[n], X) \cong$ $X_{n}$, assigning to a simplicial map $\varphi: \Delta[n] \longrightarrow X$, the image of the fundamental simplex, $\varphi\left(\iota_{n}\right) \in X_{n}$. We call $\varphi$ the characteristic map of $x$. We will often identify a simplex $x \in X_{n}$ with its characteristic map $\Delta[n] \stackrel{x}{\longrightarrow} X$, denoted with the same symbol.

Given a face $d_{i} \imath_{n} \in \Delta[n]$, the characteristic map is denoted $d^{i}: \Delta[n-1] \longrightarrow \Delta[n]$. Likewise, given a degeneracy $s_{i}\left(\imath_{n}\right)$, we have a characteristic map $s^{i}: \Delta[n+1] \longrightarrow \Delta[n]$. It turns out that the set of maps $d^{i}$ and $s^{i}, 0 \leq n, 0 \leq i \leq n$, generate all maps $\Delta[m] \rightarrow \Delta[n], n, m \geq 0$, subject to the dual relations (11). In particular, we can identify $\Delta$ with the full subcategory of $\mathbf{S}$ with objects $\Delta[n], n \geq 0$. Then, given an arbitrary simplicial set $X$, the overcategory $\Delta \downarrow X$ might be seen as an index category for the simplices of $X$ and we can express $X$ as colimit of its simplices (cf. [10, I.2.1])

$$
X \cong \operatorname{colim}_{\Delta \downarrow X} \Delta[n]
$$

We will now describe how these considerations extend to the category of $\mathcal{C}$-diagrams, for a fixed small index category $\mathcal{C}$. Then, we will also explain how the category of $\mathcal{C}$-diagrams inherits a cofibrantly generated simplicial model category structure from that of $\mathbf{S}$. We mainly follow [12] (in particular [12, 11.6.1]) but at the same time we will introduce the convenient notation for next sections.

Given a small category $\mathcal{C}$, we denote by $\mathbf{S}^{\mathcal{C}}$ the category of $\mathcal{C}$-diagrams, namely, the category of functors from $\mathcal{C}$ to $\mathbf{S}$ and natural transformations between them. Any simplicial set $X$ might be considered as a constant $\mathcal{C}$-diagram, namely a diagram where any object of $\mathcal{C}$ maps to $X$ and any morphism maps to the identity of $X$.

Let $c$ be an object of $\mathcal{C}$ and $\imath_{c}:\{c\} \rightarrow \mathcal{C}$ the inclusion of the subcategory with a unique object $c$ and a unique morphism $i d_{c}$. Given a simplicial set $Y$, we will equally denote by $Y$ the functor defined on $\{c\}$ that assigns $Y$ to $c$. Then its left Kan extension $\imath_{c, *} Y$ along the inclusion functor is a $\mathcal{C}$-diagram that can be described on objects as

$$
\imath_{c, *} Y(d)=Y \times \operatorname{Mor}_{\mathcal{C}}(c, d), \quad d \in \mathrm{Ob}(\mathcal{C})
$$

and for a morphism $f \in \operatorname{Mor}_{\mathcal{C}}(a, b)$, the induced map $\imath_{c, *} Y(f): \imath_{c, *} Y(a) \longrightarrow \imath_{c, *} Y(b)$ is defined as $\imath_{c, *} Y(f)(y, g)=(y, f \circ g)$, for each simplex $y$ of $Y$ and $g \in \operatorname{Mor}_{\mathcal{C}}(c, a)$. (cf. [12, 11.5.25]). Furthermore, there is an adjunction

$$
\operatorname{Mor}_{\mathbf{S}^{\mathcal{C}}}\left(\imath_{c, *} Y, X\right) \cong \operatorname{Mor}_{\mathbf{S}}(Y, X(c)) .
$$

for each object $c$ of $\mathcal{C}$.

Recall that the boundary of $\Delta[n]$ is defined as the smallest simplicial subset $\dot{\Delta}[n]$ of $\Delta[n]$ containing the faces $d_{i} \imath_{n}, 0 \leq i \leq n$, while the $k^{\text {th }}$-horn $\Lambda^{k}[n](0 \leq k \leq n, n \geq 1)$ is the smallest simplicial subset $\Delta[n]$ containing all of the faces $d_{i} \imath_{n}, i \neq k$. Now, we extend these definitions to the category of $\mathbf{S}^{\mathcal{C}}$. 
Definition 2.1. Let $\mathcal{C}$ be a small category. For each object $c$ of $\mathcal{C}$ we set

$$
\begin{aligned}
\delta_{n}^{c} & =\imath_{c, *} \Delta[n] \\
\dot{\delta}_{n}^{c} & =\imath_{c, *} \dot{\Delta}[n] \\
\lambda_{n, k}^{c} & =\imath_{c, *} \Lambda^{k}[n]
\end{aligned}
$$

for $n \geq 0$ and $0 \leq k \leq n$.

Definition 2.2. Let $\Gamma_{\mathcal{C}}$ be the full subcategory of $\mathbf{S}^{\mathcal{C}}$ with objects $\delta_{n}^{c}$, with $c$ in $\mathcal{C}$ and all $n \geq 0$. We define the category $\Gamma_{\mathcal{C}}^{\text {op }}$-sets as the category of functors from $\Gamma_{\mathcal{C}}^{o p}$ to the category of sets, and natural transformation between them.

By naturality of the left Kan extension, we have morphisms in $\Gamma_{\mathcal{C}}$,

$$
\begin{aligned}
& s^{i}: \delta_{n+1}^{c} \longrightarrow \delta_{n}^{c} \\
& d^{i}: \delta_{n-1}^{c} \longrightarrow \delta_{n}^{c}
\end{aligned}
$$

for all $c \in \mathrm{Ob}(\mathcal{C}), n \geq 0$ and $0 \leq i \leq n$, which are in turn induced by the simplicial maps $s^{i}: \Delta[n+1] \longrightarrow \Delta[n]$ and $d^{i}: \Delta[n-1] \longrightarrow \Delta[n]$, respectively. For every $f \in \operatorname{Mor}_{\mathcal{C}}(a, b)$ and $n \geq 0$ there is also a morphism

$$
\delta_{n}^{f}: \delta_{n}^{b} \longrightarrow \delta_{n}^{a}
$$

defined by $\left(\delta_{n}^{f}\right)_{c}(z, h)=(z, h \circ f)$, for any $c \in \mathrm{Ob}(\mathcal{C})$, any simplex $z \in \Delta[n]$ and any morphism $h \in \operatorname{Mor}_{\mathcal{C}}(b, c)$.

Proposition 2.3. All morphisms of $\Gamma_{\mathcal{C}}$ are compositions of the form $\delta_{m}^{f} d^{k_{r}} \ldots d^{k_{1}} s^{j_{1}} \ldots s^{j_{t}}$.

Proof. Take an arbitrary morphism $\eta: \delta_{n}^{c} \longrightarrow \delta_{m}^{d}$ of $\Gamma_{\mathcal{C}}$. The adjoint map is a map of simplicial sets

$$
\tilde{\eta}: \Delta[n] \longrightarrow\left(\delta_{m}^{d}\right)_{c}=\Delta[m] \times \operatorname{Hom}_{\mathcal{C}}(d, c)=\coprod_{\operatorname{Hom}_{\mathcal{C}}(d, c)} \Delta[m]
$$

Since the simplex $\Delta[n]$ is connected, the image $\widetilde{\eta}(\Delta[n])$ is contained in one of the components of $\left(\delta_{m}^{d}\right)_{c}$. Hence, we can factor $\tilde{\eta}$ as a map $\varphi: \Delta[n] \rightarrow \Delta[m]$ followed by the inclusion $\imath_{f}: \Delta[m] \longrightarrow \Delta[m] \times \operatorname{Hom}_{\mathcal{C}}(d, c)$ defined by $\imath_{f}(z)=(z, f)$. It is known that every map $\Delta[n] \longrightarrow \Delta[m]$ is a composition of face and degeneracy maps $\varphi=d^{k_{r}} \ldots d^{k_{1}} s^{j_{1}} \ldots s^{j_{t}}$. So, $\widetilde{\eta}$ is the composition shown in the left hand diagram
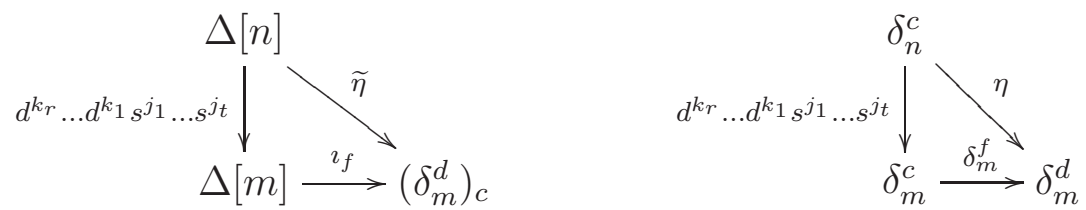

Taking adjoints we obtain the right hand diagram, and thus $\eta=\delta_{m}^{f} d^{k_{r}} \ldots d^{k_{1}} s^{j_{1}} \ldots s^{j_{t}}$.

According to Proposition 2.3, a $\Gamma_{\mathcal{C}}^{\mathrm{op}}$-set $X$ can be described as a family of sets $\left\{X_{c, n}\right\}$ indexed by objects $c$ of $\mathcal{C}$ and natural numbers $n \in \mathbb{N}$ together with structural maps

$$
\begin{array}{ll}
d_{i}: X_{c, n} \longrightarrow X_{c, n-1}, & 0 \leq i \leq n, n \geq 1, c \in \mathrm{Ob}(\mathcal{C}) \\
s_{i}: X_{c, n} \longrightarrow X_{c, n+1} & 0 \leq i \leq n, n \geq 0, c \in \mathrm{Ob}(\mathcal{C}) \\
f: X_{c, n} \longrightarrow X_{d, n} & f \in \operatorname{Mor}_{\mathcal{C}}(c, d), n \geq 0,
\end{array}
$$

satisfying the simplicial relations (11) and the naturality relations

$$
f \circ d_{i}=d_{i} \circ f \text {, and } f \circ s_{i}=s_{i} \circ f \text {, for all } f \text { and } i \text {. }
$$


Proposition 2.4. Given a small category $\mathcal{C}$, there is a natural isomorphism of categories

$$
\Gamma_{\mathcal{C}}^{\text {op }} \text {-sets } \stackrel{\cong}{\longrightarrow} \mathbf{S}^{\mathcal{C}}
$$

Proof. A $\Gamma_{\mathcal{C}}^{\text {op }}$-set $X$ is mapped to a $\mathcal{C}$-diagram $\tilde{X}$ where $\tilde{X}(c)$ is the simplicial set with $n$-simplices $X_{c, n}$, face maps $d_{i}: X_{c, n} \longrightarrow X_{c, n-1}, 1 \leq n, 0 \leq i \leq n$ and degeneracy maps $s_{i}: X_{c, n} \longrightarrow X_{c, n+1}, 0 \leq n, 0 \leq i \leq n$. We assign the map $\tilde{X}(f): \widetilde{X}(a) \longrightarrow \widetilde{X}(b)$ induced by the structural maps of $X, \delta_{n}^{f}, n \geq 0$, to every morphism $f \in \operatorname{Mor}_{\mathcal{C}}(a, b)$.

The inverse functor assigns to a $\mathcal{C}$-diagram $Y$ the $\Gamma_{\mathcal{C}}^{\text {op }}$-set $\bar{Y}$ given by $\bar{Y}\left(\delta_{n}^{c}\right)=\operatorname{Mor}_{\mathbf{S}^{c}}\left(\delta_{n}^{c}, Y\right) \cong$ $\operatorname{Hom}_{\mathbf{S}}(\Delta[n], Y(c)) \cong Y(c)_{n}$, with structural maps induced by those of each simplicial set $Y(c), c \in \mathrm{Ob}(\mathcal{C})$, and by $\delta_{n}^{f}: \delta_{n}^{b} \longrightarrow \delta_{n}^{a}$, for each $n \geq 0$, and each $f \in \operatorname{Mor}(\mathcal{C})$.

We will use this result as an identification of the categories $\mathbf{S}^{\mathcal{C}}$ and $\Gamma_{\mathcal{C}}^{\mathrm{op}}$-sets. In particular, by a $\mathcal{C}$-diagram of simplicial sets we mean an object of either of these categories, and we will choose the most useful description in each situation.

When a $\mathcal{C}$-diagram $X$ is viewed as a $\Gamma_{\mathcal{C}}^{\text {op }}$-set, every element of every set $X_{c, n}=X\left(\delta_{n}^{c}\right)$ is called a simplex, and identifying $X_{c, n} \cong \operatorname{Mor}_{\Gamma_{\mathcal{C}}^{\mathrm{op}} \text {-sets }}\left(\delta_{n}^{c}, X\right)$, we equally denote with the same letter a simplex $x \in X_{c, n}$ and its characteristic map $\delta_{n}^{c} \stackrel{x}{\longrightarrow} X$, as we do for simplicial sets. In this case we can also decompose a $\mathcal{C}$-diagram $X$ as colimit of its simplices

$$
X \cong \operatorname{colim}_{\Gamma_{\mathcal{C}} \downarrow X} \delta_{n}^{c}
$$

We will now explain the basic concepts and definitions that endow the category of $\mathcal{C}$ diagrams with the structure of a cofibrantly generated simplicial model category, extending that of $\mathbf{S}$.

Definition 2.5. A map of $\mathcal{C}$-diagrams $p: X \rightarrow B$ is a fibration if for every diagram

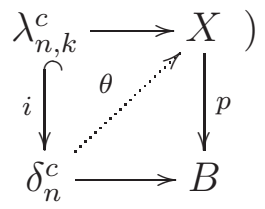

where the solid arrows commute, there is a map $\theta$, the dotted arrow, making both triangles commutative.

A $\mathcal{C}$-diagram $X$ is fibrant provided the unique map $X \longrightarrow *$ from $X$ to the constant diagram with value a point is a fibration.

This reduces to the classical definition of Kan fibration when the index category $\mathcal{C}$ is the trivial category with one object and one morphism. It clearly holds that a map of diagrams $X \longrightarrow B$ is a fibration if it restricts to a Kan fibration $X(c) \longrightarrow B(c)$ of every object $c \in \mathrm{Ob}(\mathcal{C})$.

Definition 2.6. We define weak equivalences of diagrams as those maps $X \longrightarrow Y$ that restrict to a weak equivalence of simplicial sets $X(c) \longrightarrow Y(c)$ for each $c \in \mathrm{Ob}(\mathcal{C})$.

Definition $2.7([\underline{6}, 2.4])$. A map $f: X \rightarrow Y$ of $\mathbf{S}^{\mathcal{C}}$ is called free, if for every object $c$ of $\mathcal{C}$ and $n \geq 0$, the map $f_{c, n}: X_{c, n} \longrightarrow Y_{c, n}$ is injective and if there exists a set $\Sigma(f)$ of simplices of $Y$ such that

(i) no simplex of $\Sigma(f)$ is in the image of $f$.

(ii) $\Sigma(f)$ is closed under degeneracy operators, and 
(iii) for every object $c$ of $\mathcal{C}, n \geq 0$, and every simplex $y \in Y_{c, n}$ which is not in the image of $f_{c, n}$, there exists a unique simplex $b \in \Sigma(f)$ and a unique map $h \in \operatorname{Mor}(\mathcal{C})$ such that $\delta_{n}^{h}(b)=y$.

We will say that a $\mathcal{C}$-diagram $X$ is free if the map $\emptyset \longrightarrow X$ from the empty diagram is free.

According to this definition a $\mathcal{C}$-diagram $X$ is free if there is a set $\Sigma=\Sigma(X)$ of simplices of $X$ which is closed under degeneracies and generates $X$ freely; namely, for each simplex $x \in X$, there is a unique $w \in \Sigma(X)$ and a unique morphism $h$ in $\mathcal{C}$, such that $\delta_{n}^{h}(w)=x$. The set $\Sigma$ is called a basis for $X$, and an element of $\Sigma$ is called a generator. We will call $\Sigma_{n}$ the subset of $\Sigma$ consisting of all $n$-simplices of $X$ that belong to $\Sigma_{n}=\Sigma \cap X_{n}$. Similarly, $\Sigma_{c}=\Sigma \cap X_{c}$ and $\Sigma_{c, n}=\Sigma \cap X_{c, n}$, for $n \geq 0$ and $c \in \mathrm{Ob}(\mathcal{C})$

In the case of a free map $f: X \longrightarrow Y$, we will also call a basis for $f$ the set $\Sigma(f)$ over which $f$ is free according to Definition 2.7 ,

Example 2.8. Let $X$ be a $\mathcal{C}$-diagram. For any $n \geq 0, c \in \mathcal{C}$, and any map $\varphi$ in $\operatorname{Mor}_{\mathbf{S}^{c}}\left(\dot{\delta}_{n}^{c}, X\right)$, the map $\imath: X \longrightarrow Y$ defined by the push-out diagram

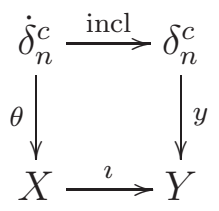

is a free map with $\Sigma(\imath)=\{y\} \cup$ iterated degeneracies of $y\}$, where $y$ is the $n$-simplex of $Y$ classified by the map $y: \delta_{n}^{c} \longrightarrow Y$. Moreover, if $X$ is free with basis $\Sigma(X)$, then $Y$ is also a free $\mathcal{C}$-diagram with $\Sigma(Y)=\Sigma(X) \cup \Sigma(\imath)$

Actually, all free maps and free $\mathcal{C}$-diagrams can be obtained by a possibly transfinite iteration of the above example. A free map between $\mathcal{C}$-diagrams, $\omega: X \rightarrow Y$ with base $\Sigma$, can easily be seen to be the composition of a sequence

$$
X \longrightarrow Y_{0} \longrightarrow Y_{1} \longrightarrow \ldots \longrightarrow Y_{i} \longrightarrow Y_{i+1} \longrightarrow \ldots=\operatorname{colim}_{i} Y_{i}
$$

where each step $Y_{i} \longrightarrow Y_{i+1}$ is produced by a pushout diagram that generalizes Example 2.8 from attaching a single simplex to the case of a collection of simplices of dimension $i+1$.

Conversely, any transfinite iteration of the construction of Example 2.8 is a free map. Actually, a standard transfinite induction argument shows that given an ordinal $\lambda$, and a $\lambda$-sequence of maps of $\mathcal{C}$-diagrams

$$
X_{0} \stackrel{\varphi_{1}}{\longrightarrow} X_{1} \stackrel{\varphi_{2}}{\longrightarrow} X_{2} \longrightarrow \ldots \stackrel{\varphi_{\beta}}{\longrightarrow} X_{\beta} \longrightarrow \ldots
$$

with $\beta<\lambda$, where any of the maps $X_{\beta} \longrightarrow X_{\beta+1}, \beta+1<\lambda$, is a free map and for any limit ordinal $\gamma<\lambda$, the induced map $\operatorname{colim}_{\beta<\gamma} X_{\beta} \longrightarrow X_{\gamma}$ is an isomorphism, we can choose basis $\Sigma_{\beta}$ for the partial compositions $\omega_{\beta}: X_{0} \longrightarrow X_{\beta}$, such that $\omega_{\alpha, \beta}\left(\Sigma_{\alpha}\right) \subseteq \Sigma_{\beta}$ if $\alpha<\beta$ and the composition

$$
X_{0} \longrightarrow \operatorname{colim}_{\beta<\lambda} X_{\beta}
$$

is a free map with basis $\Sigma_{\lambda}=\operatorname{colim}_{\beta<\lambda} \Sigma_{\beta}$.

This shows in particular that in the category of $\mathcal{C}$-diagrams the notion of free maps and free $\mathcal{C}$-diagrams of [6], as stated in Definition [2.7, coincides with that of relative free cell complex and free cell complex in [12, 11.5.35]. This justifies the next definition (cf. [6, 2.4], [12, 11.6.1]).

Definition 2.9. A map of $\mathcal{C}$-diagrams is called a cofibration if it is a retract of a free map. Likewise, a $\mathcal{C}$-diagram is cofibrant if it is a retract of a free diagram. 
If the index category is the trivial category with one object and one morphism, then the concept of cofibration reduces to the classical notion for simplicial sets, where a map is a cofibration if it is injective. If we have a category with two objects $a$ and $b$ and a unique non-identity morphism $f: a \longrightarrow b$, then a $\mathcal{C}$-diagram $X$ is free if and only if the map $X(f)$ is injective.

Given a discrete group $G$, a simplicial set $X$ with an action of $G$ can be seen as a diagram defined over the category $\mathcal{B} G$ with one object and the elements of $G$ as morphisms. This is a free diagram if and only if $G$ acts freely on $X$.

For an arbitrary small category $\mathcal{C}$, if $Y$ is a simplicial set and $c$ is an object of $\mathcal{C}$, the $\mathcal{C}$-diagram $\imath_{c, *} Y$ is a free diagram on generators $\Sigma=Y \times\left\{\operatorname{Id}_{c}\right\} \subseteq \imath_{c, *} Y(c)=Y \times$ $\operatorname{End}_{\mathcal{C}}(c)$. Likewise, if $f: A \longrightarrow B$ is an inclusion of simplicial sets, then the induced map $\imath_{c, *} f: \imath_{c, *} A \longrightarrow \imath_{c, *} B$ is a free map. This applies in the next definition:

Definition 2.10. Let $\mathcal{C}$ be a small category. The generating cofibrations of $\mathbf{S}^{\mathcal{C}}$ are the maps

$$
\dot{\delta}_{n}^{c} \longrightarrow \delta_{n}^{c}, \quad \text { for all } c \in \mathrm{Ob}(\mathcal{C}), n \geq 0,
$$

induced by the inclusions $\dot{\Delta}[n] \hookrightarrow \Delta[n]$.

The generating trivial cofibrations are the maps

$$
\lambda_{n, k}^{c} \hookrightarrow \delta_{n}^{c}, \quad \text { for all } c \in \mathrm{Ob}(\mathcal{C}), n>0 \text {, and } 0 \leq k \leq n,
$$

induced by the inclusions $\Lambda^{k}[n] \hookrightarrow \Delta[n]$.

The category of $\mathcal{C}$-diagrams also inherits a simplicial model category structure from the category of simplicial sets, with structure natural functors, external product, function complex and exponent functor. We will briefly recall these constructions. The external product with simplicial sets is defined

$$
\times: \Gamma_{\mathcal{C}}^{\mathrm{op}} \text {-sets } \times \mathbf{S} \longrightarrow \Gamma_{\mathcal{C}}^{\mathrm{op}} \text {-sets }
$$

defined by $(X \times K)_{c, n}=X_{c, n} \times K_{n}$, and with structural maps induced by those of $X$ and $K$. Notice that we can similarly define a left product with simplicial sets. We use both form without further explanation.

Definition 2.11. The function complex

$$
\operatorname{map}_{\mathcal{C}}: \Gamma_{\mathcal{C}}^{\mathrm{op}} \text {-sets } \times \Gamma_{\mathcal{C}}^{\mathrm{op}} \text {-sets } \longrightarrow \mathbf{S}
$$

is defined, for two $\mathcal{C}$-diagrams $X$ and $Y$, as the $\operatorname{simplicial}$ set $\operatorname{map}_{\mathcal{C}}(X, Y)$ with $n$-simplices $\operatorname{Mor}_{\mathbf{S}^{\mathcal{C}}}(X \times \Delta[n], Y)$ or equivalently, the commutative diagrams in $\mathbf{S}^{\mathcal{C}}$

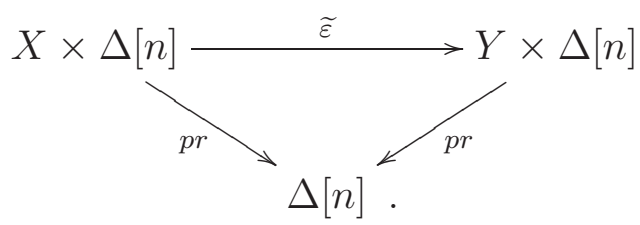

Now, if $g \in \operatorname{Mor}_{\mathbf{S}}(\Delta[n] \times X, Y)$, its faces $d_{i} g$ and degeneracies $s_{i} g$ are given by the compositions

$$
\Delta[n-1] \times X \stackrel{d^{i} \times 1}{\longrightarrow} \Delta[n] \times X \stackrel{g}{\longrightarrow} Y \quad \text { and } \quad \Delta[n+1] \times X \stackrel{s^{i} \times 1}{\longrightarrow} \Delta[n] \times X \stackrel{g}{\longrightarrow} Y .
$$

Finally, there is an exponent functor and

$$
\wedge: \mathbf{S} \times \Gamma_{\mathcal{C}}^{\mathrm{op}} \text {-sets } \longrightarrow \mathbf{S}
$$

with $X^{K}=\operatorname{map}_{\mathcal{C}}(K, X)$, where $K$ is the constant $\mathcal{C}$-diagram. 
With the above definitions, the basic theory of simplicial sets extends to $\mathcal{C}$-diagrams. Indeed, $\mathbf{S}^{\mathcal{C}}$ becomes a cofibrantly generated simplicial model category [12, 11.6.1, 11.7.3]. In the next definition we collect the axioms for later reference.

Definition 2.12 ([12, 7.1.3, 9.1.6, and 11.1.2]). Let $\mathcal{M}$ be a category equipped with three classes of morphisms called fibrations, cofibrations, and weak equivalences. A fibration (respectively cofibration) which is also a weak equivalence is called a trivial fibration (respectively trivial cofibration). Then, $\mathcal{M}$ is a model category [16] if it satisfies the following axioms:

M1: The category $\mathcal{M}$ is closed under small limits and colimits.

M2: Given composable maps $X \stackrel{g}{\longrightarrow} Y \stackrel{f}{\longrightarrow} Z$ in $\mathcal{M}$, if any two of $f, g$ and $f \circ g$ are weak equivalences, then so is the third.

M3: If $f$ is a retract of $g$ and $g$ is a weak equivalence, fibration or cofibration, then so is $f$.

M4: Suppose that we are given a commutative solid arrow diagram

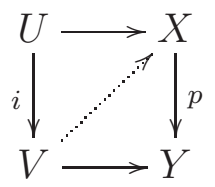

where $i$ is a cofibration and $p$ is a fibration. If either $i$ is a trivial cofibration or $p$ is a trivial fibration, then the dotted arrow exists and makes the diagram commutative.

M5: Every map $f$ of $\mathcal{M}$ has two functorial factorizations:

(a) $f=p \circ i$ where $p$ is a fibration and $i$ is a trivial cofibration, and

(b) $f=q \circ j$ where $q$ is a trivial fibration and $j$ is a cofibration.

A model category $\mathcal{M}$ is said to be cofibrantly generated if

(1) There exists a set $I$ of maps (called a set of generating cofibrations) that permits the small object argument $([12,10.5 .15])$ and such that a map is a trivial fibration if and only if it has the $R L P$ with respect to every element of $I$.

(2) There exists a set $J$ of maps (called a set of trivial generating cofibrations) that permits the small object argument and such that a map is a fibration if and only if it has the $R L P$ with respect to every element of $J$.

If $\mathcal{M}$ is a model category, then it is a simplicial model category if it is enriched over simplicial sets, and the following two axioms hold:

M6: For every two objects $X$ and $Y$ of $\mathcal{M}$ and every simplicial set $K$ there exist objects $X \otimes K$ and $Y^{K}$ of $\mathcal{M}$ such that there are isomorphisms of simplicial sets

$$
\operatorname{hom}(X \otimes K, Y) \cong \operatorname{hom}(K, \operatorname{hom}(X, Y)) \cong \operatorname{hom}\left(X, Y^{K}\right)
$$

that are natural in $X, Y$ and $K$.

M7: If $i: A \longrightarrow B$ is a cofibration in $\mathcal{M}$ and $p: X \rightarrow Y$ is a fibration in $\mathcal{M}$, then the map of simplicial sets

$$
\left(i^{*}, p_{*}\right): \operatorname{hom}(B, X) \longrightarrow \operatorname{hom}(A, X) \times_{\operatorname{hom}(A, Y)} \operatorname{hom}(B, Y)
$$

is a fibration that is a trivial fibration if either $i$ or $p$ is a weak equivalence.

Remark 2.13. From the cofibrantly generated model structure for $\mathbf{S}^{\mathcal{C}}$ and Quillen Small Object Argument ([16], [12, 10.5.16]), we obtain that any map $\emptyset \rightarrow X$ in $\mathbf{S}^{\mathcal{C}}$ admits a functorial factorization $\emptyset \hookrightarrow Q X \stackrel{\sim}{\longrightarrow} X$, where $Q X$ is free and the first map is a cofibration while the second map is a trivial fibration [12, 10.5.2, 11.2.1-1]. 
The following consequence of the axioms will be useful in later sections. The proof can be seen in [12, 9.3.8].

Proposition 2.14. Let $\mathcal{M}$ be a simplicial model category. It $i: A \longrightarrow B$ is a cofibration, and $K \subseteq L$ simplicial sets, the induced map

$$
A \otimes L \coprod_{A \otimes K} B \otimes K \longrightarrow B \otimes L
$$

is also a cofibration and it is a trivial cofibration if either $i: A \longrightarrow B$ is a trivial cofibration or the inclusion $K \subseteq L$ is a weak equivalence.

Corollary 2.15. The map $\dot{\delta}_{n}^{c} \times \Delta[m] \cup \delta_{n}^{c} \times \Lambda^{k}[m] \hookrightarrow \delta_{n}^{c} \times \Delta[m]$ is a trivial cofibration.

\section{Minimal Fibrations of DiagRAms of SimpliCial SETS}

In this section we develop a theory of minimal fibrations in the context of diagrams of simplicial sets, that generalizes the classical theory for simplicial sets [1, 9, 10, 13, 15]. We will show that under some restrictions on the shape of the index category $\mathcal{C}$, the theory of minimal fibrations carries out to the case of $\mathcal{C}$-diagrams. We prove Theorem $\mathrm{A}$ that gives sufficient conditions under which a fibration of $\mathcal{C}$-diagrams is fibrewise equivalent to a minimal fibration. Corollary 3.12 shows that two fibrewise homotopic minimal fibrations are unique up to isomorphism, thus the associated minimal fibration of Theorem $\mathrm{A}$ is unique up to isomorphism. Recall that a fibre homotopy equivalence between fibrations with the same base space $B$ is a map over $B$ that admits a homotopy inverse, also over $B$. It is understood that the homotopies defining the homotopy inverse are also maps over $B$.

The concept of minimal fibration in this context is defined below, in Definition 3.3. This is somewhat technical but it extends the classical definition and it seems to be the appropriate choice to the arguments that follow. We will show that under some restrictions on the shape of the index category every fibration with free total $\mathcal{C}$-diagram admits a strong fibrewise deformation retract which is a minimal fibration and unique up to isomorphism (Theorem $\mathrm{A}$ and Corollary 3.12). Under the same restrictions, Lemma 3.9] gives an alternative characterization of minimality that could be used as a more intrinsic definition of the same concept.

Definition 3.1. If $\mathcal{C}$ is a small category and $p: X \longrightarrow B$ a fibration of $\mathcal{C}$-diagrams, we say that two simplices $x, y \in X_{c, n}$ are $p$-homotopic, $x \simeq_{p} y$, if there is a fibrewise homotopy from $x$ to $y$ relative to the boundary; that is, there is a homotopy

$$
H: \delta_{n}^{c} \times \Delta[1] \longrightarrow X,
$$

such that

(i) $H_{0}: \delta_{n}^{c} \longrightarrow X$ is the characteristic map of the simplex $x$ and $H_{1}: \delta_{n}^{c} \longrightarrow X$ is the characteristic map of the simplex $y$,

(ii) $\left.H\right|_{\dot{\delta}_{n}^{c} \times \Delta[1]}: \dot{\delta}_{n}^{c} \times \Delta[1] \longrightarrow X$ is a constant homotopy,

(iii) $p \circ H: \delta_{n}^{c} \times \Delta[1] \longrightarrow B$ is a constant homotopy.

Definition 3.2. Let $\mathcal{C}$ be a small category and $p: X \longrightarrow B$ a fibration of $\mathcal{C}$-diagrams. We will say that a simplex $y \in X$ is sub-p-homotopic to $x$, or that $x$ precedes $y, x \preceq y$, if there exists a morphisms $f \in \operatorname{Mor}(\mathcal{C})$ such that $f(x) \simeq_{p} y$. We will write:

$$
[x,-) \stackrel{\text { def }}{=}\{y \in X \mid x \preceq y\} \text {. }
$$

The following definition generalizes the concept of minimal fibration of simplicial sets ([11], [15, 10.1]). 
Definition 3.3. Let $\mathcal{C}$ be a small category. A fibration $X \longrightarrow B$ in $\mathbf{S}^{\mathcal{C}}$ is said to be minimal if $X$ is free and given any two generators $x, y$ of a base $\Sigma$ of $X, x \preceq y$ implies $x=y$. An object $X$ of $\mathbf{S}^{\mathcal{C}}$ is called minimal if the map $X \longrightarrow *$ is a minimal fibration.

The next lemma shows that if the condition of this definition holds for any one base then it holds for every other base.

Lemma 3.4. Let $\mathcal{C}$ be a small category and $p: X \longrightarrow B$ a fibration of $\mathcal{C}$-diagrams where $X$ is a free $\mathcal{C}$-diagram. If there is a base $\Sigma$ of $X$ such that given simplices $x, y \in \Sigma, x \preceq y$ implies $x=y$, then the same holds for any other base of $X$.

Proof. Assume that $\Sigma^{\prime}$ is a different base set for $X$. Then for any $x \in \Sigma^{\prime}$ there is a simplex $u \in \Sigma$ and an isomorphism $f \in \operatorname{Iso}(\mathcal{C})$ with $f(u)=x$. Since $\Sigma$ is a base, given $x \in \Sigma$, there is a unique $u \in \Sigma^{\prime}$ and a unique morphism $f \in \operatorname{Mor}(\mathcal{C})$ such that $f(u)=x$. But $\Sigma^{\prime}$ is a base, so there is $y \in \Sigma^{\prime}$ and $g \in \operatorname{Mor}(\mathcal{C})$ such that $g(y)=u$. Then $(f \circ g)(y)=x$, but both $y$ and $x$ are in $\Sigma^{\prime}$, and hence $x=y$ and $f \circ g$ is the identity morphism on the source of $g$. Now we also have $(g \circ f)(u)=u$ and $u \in \Sigma$, so $g \circ f$ is also the identity over the source of $f$. Thus $f$ is an isomorphism of $\mathcal{C}$ with $f^{-1}=g$.

We can now conclude that $\Sigma^{\prime}$ satisfies the same condition as $\Sigma$. Assume that $x, y \in \Sigma^{\prime}$ satisfy that $x \preceq y$, that is, there is $\varphi \in \operatorname{Mor}(\mathcal{C})$ such that $\varphi(x) \simeq_{p} y$. We will conclude that $x=y$. There are $u, v$ in $\Sigma$ and isomorphisms $f, g \in \operatorname{Iso}(\mathcal{C})$, such that $f(u)=x$ and $g(v)=y$. It follows that $g^{-1}(\varphi(f(u)))=g^{-1}(\varphi(x)) \simeq_{p} g^{-1}(y)=v$, so $u \preceq v$ and, by the assumption on $\Sigma, u=v$. Then, $\left(f \circ g^{-1}\right)(y)=x$, but both $x$ and $y$ are in the base set $\Sigma^{\prime}$, so we have $x=y$.

Let $\mathcal{C}$ be a small category. Given $a, b \in \mathrm{Ob}(\mathcal{C})$, we declare $a \sim b$ provided that there are morphisms $a \longrightarrow b$ and $b \longrightarrow a$ in $\mathcal{C}$. This is an equivalence relation and the set of equivalence classes $\mathrm{Ob}(\mathcal{C}) / \sim$ becomes a poset with the order relation defined by $[a] \leq[b]$ if and only if there is an arrow $a \longrightarrow b$ in $\mathcal{C}$. Notice that the definition of $\leq$ does not depend on the choice of representatives in the classes $[a]$ and $[b]$.

Definition 3.5. We will say that a small category $\mathcal{C}$ is artinian if it satisfies the descending chain condition on the poset $(\mathrm{Ob}(\mathcal{C}) / \sim, \leq)$. Namely, if every descending chain $\left[a_{1}\right] \geq\left[a_{2}\right] \geq$ $\left[a_{3}\right] \geq \ldots$ in $\mathrm{Ob}(\mathcal{C}) / \sim$ eventually stabilizes.

Definition 3.6. Let $X$ be a $\mathcal{C}$-diagram in $\mathbf{S}$. A subdiagram of $X$ consists of a subset $X^{\prime}$ of simplices of $X$ which is in itself a $\mathcal{C}$-diagram. This relation is denoted by $X^{\prime} \leq X$.

The following is a classical notion (see for example [14]):

Definition 3.7. An $E I$-category is a category $\mathcal{C}$ in which every endomorphism is an isomorphism.

We need to introduce some notation now.

Notation 3.8. Since $\Lambda^{k}[n]$ is the subcomplex of $\Delta[n]$ generated by all of the faces $d_{i} \imath_{n}$ of $\Delta[n]$, except for the $k$ th face $d_{k} \imath_{n}$, a map $\alpha: \Lambda^{k}[n] \longrightarrow X$ is thus determined by the restrictions to that faces. Accordingly, we will denote

$$
\alpha=\left(x_{0}, \ldots, x_{k-1},-, x_{k+1}, \ldots, x_{n}\right),
$$

where $x_{i}=\alpha\left(d_{i} \imath_{n}\right)$ is the simplex of $X$ characterized by the restriction of $\alpha$ to the $i$ th face. The analogue notation $\alpha=\left(x_{0}, \ldots, x_{k-1}, x_{k}, \ldots, x_{n}\right)$ will be used for maps $\alpha: \dot{\Delta}[n] \longrightarrow X$, except that there are no blanks in this case. 
More generally, a map $\varphi: Z \times \Lambda^{k}[n] \longrightarrow X$ is written

$$
\varphi=\left(\alpha_{0}, \ldots, \alpha_{k-1},-, \alpha_{k+1}, \ldots, \alpha_{n}\right) \text {, }
$$

where for each $i \neq k, \alpha_{i}$ is the composition $Z \times \Delta[n-1] \stackrel{\operatorname{Id}_{Z} \times d^{i}}{\longrightarrow} Z \times \Lambda^{k}[n] \stackrel{\varphi}{\longrightarrow} X$.

These same conventions extend to the case of $\mathcal{C}$-diagrams. If $X$ is a $\mathcal{C}$-diagram, a map $u: \dot{\delta}_{c}^{n} \longrightarrow X$ is written $u=\left(u_{0}, \ldots, u_{n}\right)$, where $u_{i}$ is the composition $\delta_{c}^{n_{1}} \stackrel{d^{i}}{\longrightarrow} \dot{\delta}_{c}^{n} \stackrel{u}{\longrightarrow} X$.

Now we are ready to prove the main result of this section. It is stated as Theorem $\mathrm{A}$ in the introduction.

Proof of Theorem $A$. Fix an artinian $E I$-category $\mathcal{C}$, a fibration $p: X \longrightarrow B$ of $\mathcal{C}$-diagrams, and assume that $X$ is a free $\mathcal{C}$-diagram. We will construct a strong fibrewise deformation retract $q: \hat{X} \longrightarrow B$, which is a minimal fibration of $\mathcal{C}$-diagrams.

Consider a basis $\Sigma$ of $X$. Let $\Sigma^{\prime}$ be a minimal subset of $\Sigma$ such that:

(1) Every element of $\Sigma$ is preceded by an element of $\Sigma^{\prime}$, that is, for every $w \in \Sigma$ there exists some $x \in \Sigma^{\prime}$ such that $x \preceq w$.

(2) $\Sigma^{\prime}$ contains degenerate representatives whenever it is possible. That is, if $x \in \Sigma^{\prime}$ and $[x,-)=[w,-)$ with $w$ a degenerate simplex of $\Sigma$, then $x$ is a degenerate simplex.

The set $\Sigma^{\prime}$ exists, since $\mathcal{C}$ is a small artinian EI-category. Proposition A.1 allows a choice of a minimal subset $\Sigma^{\prime}$ satisfying condition (1), and according to Remark A.2 we can refine the choice so that condition (2) is also satisfied.

Step 1. $\Sigma^{\prime}$ is closed under degeneracy operators.

Choose an $n$-simplex $w \in \Sigma^{\prime}$. Given a degeneracy operator $s_{k}, k=0, \ldots, n$, we must show that $s_{k}(w) \in \Sigma^{\prime}$.

Note that $\Sigma$ is closed under degeneracy operators, so $s_{k} w$ belongs to $\Sigma_{n+1}$. Then, there exists a simplex $x \in \Sigma_{n+1}^{\prime}$ such that $x \preceq s_{k}(w)$. There also exists a map $f \in \operatorname{Mor}(\mathcal{C})$ such that $f(x) \simeq_{p} s_{k} w$, so in particular $d_{k} f(x)=d_{k} s_{k}(w)$, and then $f\left(d_{k} x\right)=w$. Since $w \in \Sigma^{\prime} \subseteq \Sigma$ there exists $g \in \operatorname{Mor}(\mathcal{C})$ such that $g(w)=d_{k} x$. By our choice of $f$ and $g, g f$ is an endomorphism, and hence an automorphism, so let $(g f)^{-1}$ be its inverse map. Now $x=(g f)^{-1} g f(x) \simeq_{p}(g f)^{-1} g\left(s_{k} w\right)$, so $s_{k} w \preceq x$. Thus, $[x,-)=\left[s_{k} w,-\right)$ and then by condition (2), $x$ is a degenerate simplex.

Now, we have two degenerate simplices $x$ and $y=s_{k}(w)$ in $\Sigma$ and one precedes the other. Since $x \preceq y$, there exists $f \in \mathcal{C}$ such that $f(x) \simeq_{p} y$. These are $p$-homotopic degenerate simplices in a simplicial set, so $f(x)=y$ [15, 9.3]; but $x$ and $y$ are in the same orbit, and then they must coincide since both belong to $\Sigma$, that is, $x=y=s_{k} w \in \Sigma^{\prime}$.

Step 2. The set $\mathfrak{A}=\left\{(\widehat{X}, \widehat{\Sigma}) \mid \widehat{X} \subseteq X, \widehat{X}\right.$ is free and $\widehat{\Sigma}$, and $\left.\widehat{\Sigma} \subseteq \Sigma^{\prime}\right\}$ contains maximal elements for the order relation defined on $\mathfrak{A}$ by inclusion, namely $(\widehat{X}, \widehat{\Sigma}) \leq(\widetilde{X}, \widetilde{\Sigma})$ if and only if both $\widehat{X} \subseteq \widetilde{X}$ and $\widehat{\Sigma} \subseteq \widetilde{\Sigma}$.

The above relation clearly defines a partial order relation on $\mathfrak{A}$. Moreover $\mathfrak{A}$ is non-empty, since it contains the pair $\left(\left\langle\Sigma_{0}^{\prime}\right\rangle, \widehat{\Sigma}_{0}^{\prime}\right)$, where $\left\langle\Sigma_{0}^{\prime}\right\rangle$ is the $\mathcal{C}$-diagram generated by all of the 0-simplices of $\Sigma^{\prime}$, and $\widehat{\Sigma}_{0}^{\prime}$ consists of all of the elements of $\widehat{\Sigma}_{0}^{\prime}$ and all their degeneracies. More precisely, $\left\langle\Sigma_{0}^{\prime}\right\rangle$ is the $\mathcal{C}$-subdiagram of $X$ whose set of $n$-simplices at any $c \in \mathcal{C}$ is

$$
\left\langle\Sigma_{0}^{\prime}\right\rangle_{c, n}=\left\{f(x) \mid x \in s_{0}^{n} \Sigma_{0}^{\prime} \text { and } f \text { is a morphism of } \mathcal{C} \text { with codomain } c\right\},
$$

and $\widehat{\Sigma}_{0}^{\prime}=\coprod_{n \geq 0} s_{0}^{n}\left(\Sigma_{0}^{\prime}\right)$. Now $\widehat{\Sigma}_{0}^{\prime} \subset \Sigma^{\prime}$ since the latter is closed under degeneracy operators. It is also clear that $\left\langle\Sigma_{0}^{\prime}\right\rangle$ is freely generated by $\widehat{\Sigma}_{0}^{\prime}$. Thus, $\left(\left\langle\Sigma_{0}^{\prime}\right\rangle, \widehat{\Sigma}_{0}^{\prime}\right) \in \mathfrak{A}$. If we take a chain 
$\left\{\left(\widehat{X}_{i}, \widehat{\Sigma}_{i}\right)\right\}_{i \in I}$ in $\mathfrak{A}$, its union $\left(\cup_{i \in I} \widehat{X}_{i}, \cup_{i \in I} \widehat{\Sigma}_{i}\right)$ is a $\mathcal{C}$-subdiagram of $X$ and it is free with base set $\cup_{i \in I} \widehat{\Sigma}_{i} \subseteq \Sigma^{\prime}$. Hence, it belongs to $\mathfrak{A}$ and it is an upper bound of the chain. Now Zorn's Lemma implies that $\mathfrak{A}$ contains maximal elements.

Step 3. Fix a maximal element $(\widehat{X}, \widehat{\Sigma})$ of $\mathfrak{A}$. If $w$ is an $n$-simplex of $X$ such that

(i) $w$ is in the orbit of an element of $\Sigma^{\prime}$, and

(ii) $d_{k}(w) \in \widehat{X}$ for each $k=0, \ldots, n$,

then $w \in \widehat{X}$.

There is $x \in \Sigma^{\prime}$ and $f \in \operatorname{Mor}(\mathcal{C})$ such that $f(x)=w$. Since $d_{k} w \in \widehat{X}$, there exists $z \in \widehat{\Sigma} \subseteq \widehat{X}$ and $g \in \operatorname{Mor}(\mathcal{C})$ such that $g(z)=d_{k} w$. In particular, $d_{k} x, d_{k} w$ and $z$ are in the same orbit of simplices of $X$. Since $z \in \widehat{\Sigma} \subseteq \Sigma$, there is $h \in \operatorname{Mor}(\mathcal{C})$ such that $h(z)=d_{k} x$, and thus $d_{k} x \in \widehat{X}$. As this is true for each $k=0, \ldots, n$, it follows that the subdiagram $\widehat{X}_{1}=\widehat{X} \cup\langle x\rangle$ of $X$ is free with base $\widehat{\Sigma}_{1}=\widehat{\Sigma} \cup\{x\} \cup\{$ iterated degeneracies of $x\} \subseteq \Sigma^{\prime}$ (see 2.8). Hence, $\left(\widehat{X}_{1}, \widehat{\Sigma}_{1}\right) \in \mathfrak{A}$ and $(\widehat{X}, \widehat{\Sigma}) \leq\left(\widehat{X}_{1}, \widehat{\Sigma}_{1}\right)$. But $(\widehat{X}, \widehat{\Sigma})$ is maximal in $(\mathfrak{A}, \leq)$, and so $\widehat{X} \cup\langle x\rangle=\widehat{X}$. Therefore $w \in \widehat{X}$, since $f(x)=w$.

Step 4. Define $\mathfrak{B}$ as the set that consists of all the pairs $(Y, H)$, where $Y$ is a subdiagram of $X$ containing $\widehat{X}$ and $H: Y \times \Delta[1] \longrightarrow X$ is a homotopy satisfying:

- $H_{0}$ maps $Y$ into $\widehat{X}$, being $H_{0}$ the composition

$$
Y \cong Y \times \Delta[0] \stackrel{1 \times d^{1}}{\longrightarrow} Y \times \Delta[1] \stackrel{H}{\longrightarrow} X
$$

- $H_{1}$ is the inclusion of $Y$ in $X$, being $H_{1}$ the composition

$$
Y \cong Y \times \Delta[0] \stackrel{1 \times d^{0}}{\longrightarrow} Y \times \Delta[1] \stackrel{H}{\longrightarrow} X
$$

- The restriction $\left.H\right|_{\widehat{X} \times \Delta[1]}$ to $\widehat{X} \times \Delta[1]$, is constant, and then equal to the composition $\widehat{X} \times \Delta[1] \stackrel{\mathrm{pr}}{\longrightarrow} \widehat{X} \stackrel{\text { incl }}{\longrightarrow} X$.

- $p \circ H$ is also constant, hence equal to the composition $Y \times \Delta[1] \stackrel{\text { pr }}{\longrightarrow} Y \stackrel{\text { incl }}{\longrightarrow} X \stackrel{p}{\longrightarrow} B$.

We can define a partial order ' $\leq$ ' over $\mathfrak{B}$ as follows: $(Y, H) \leq\left(Y^{\prime}, H^{\prime}\right)$ if $Y \subseteq Y^{\prime}$ and $\left.H^{\prime}\right|_{Y \times \Delta[1]}=H$. The diagram $\widehat{X}$ with the constant homotopy belongs to $\mathfrak{B}$, and so $\mathfrak{B} \neq \emptyset$. Moreover, if we take a chain $\left\{\left(Y_{i}, H_{i}\right)\right\}_{i \in I}$ of $\mathfrak{B}$, its colimit $\left(Y_{\infty}, H_{\infty}\right)$, given by $Y_{\infty}=\bigcup_{i \in I} Y_{i}$ and $H_{\infty}(x, t)=H_{i}(x, t)$ if $x \in Y_{i}$, is an element of $\mathfrak{B}$, which is in turn an upper bound of the chain in $\mathfrak{B}$. Then $\mathfrak{B}$ has maximal elements again by Zorn's lemma.

Step 5. If $(Y, H)$ is maximal in $\mathfrak{B}$, then $Y=X$.

Assume that $Y \neq X$ and choose a simplex $z \in X$ of lowest dimension such that $z \notin Y$. It is non-degenerate (otherwise it would belong to $Y$ ) and its faces belong to $Y$. Assuming $z \in X_{d, n}$, let $z: \delta_{n}^{d} \longrightarrow X$ be the map classified by $z$. Then, the restriction to the boundary of $\delta_{n}^{d}$ factors through $Y$, so we denote by $\dot{z}: \dot{\delta}_{n}^{d} \longrightarrow Y$ the restriction. Then, $Y \subseteq Y \cup_{\dot{z}} \delta_{n}^{d} \subseteq$ $X$. We will extend the homotopy $H$ to $Y \cup_{\dot{z}} \delta_{n}^{d}$.

To this aim, we first use the homotopy $H$ to find a new simplex, fibrewise homotopic to $z$ (though not relative to boundary), with boundary inside $\widehat{X}$. Since $H \circ(\dot{z} \times 1): \dot{\delta}_{n}^{d} \times \Delta[1] \longrightarrow X$ coincides with $z: \delta_{n}^{d} \times \Delta[1] \longrightarrow X$ in the intersection $\dot{\delta}_{n}^{d} \times\{1\}$ 
we have a solid arrow commutative diagram

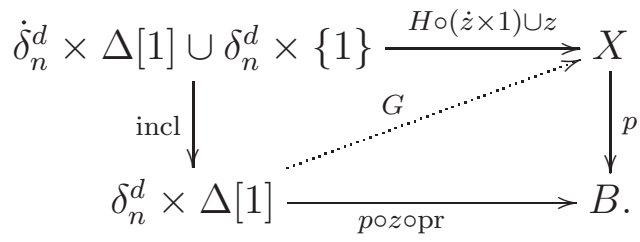

As the left vertical map is a trivial cofibration (see 2.15), according to the homotopy lifting property there is a homotopy $G$ that makes the whole diagram commutative. Notice that $G_{1}=z$, while $G_{0}$ determines another simplex $z_{1} \in X$ with boundary $\dot{z}_{1} \in \widehat{X}$. The restriction to the boundary is $\left.G\right|_{\dot{\delta}_{n}^{d} \times \Delta[1]}=H \circ(\dot{z} \times 1)$.

Now there exists a simplex $x \in \Sigma^{\prime}$ that precedes $z_{1}, x \preceq z_{1}$, such that there exists $f \in \operatorname{Mor}(\mathcal{C})$ with $y=f(x) \simeq_{p} z_{1}$. Since $\dot{x}=\dot{z}_{1} \in \widehat{X}$, Step 3 implies that $y \in \widehat{X}$.

We now combine the homotopy $G$ with a chosen fibrewise homotopy $F$ between $z_{1}$ and $y$, relative to boundary. This is achieved by means of the homotopy lifting property applied to the solid arrow commutative diagram

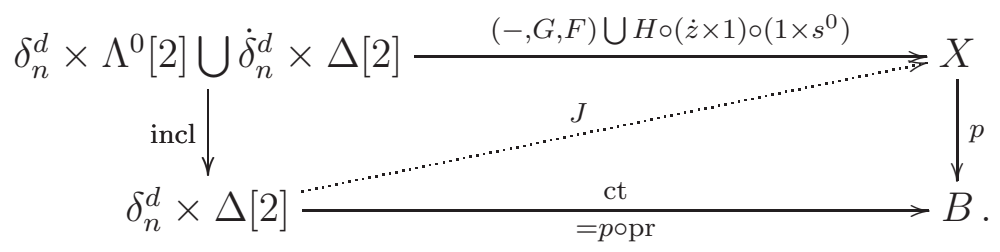

Let $J_{0}$ be the restriction of $J$ to the zero edge, namely, the composition

$$
\delta_{n}^{d} \times \Delta[1] \stackrel{1 \times d^{0}}{\longrightarrow} \delta_{n}^{d} \times \Delta[2] \stackrel{J}{\longrightarrow} X .
$$

Then, $J_{0}$ is a fibrewise homotopy from $y$ to $z$, with restriction to the boundary $\left.J_{0}\right|_{\dot{\delta}_{n}^{d} \times \Delta[1]}=$ $H \circ(\dot{z} \times 1)$. This equality makes possible an extension $\widetilde{H}:\left(Y \cup_{\dot{z}} \delta_{n}^{d}\right) \times \Delta[1] \longrightarrow X$ of $H$, that is defined by the push-out diagram:

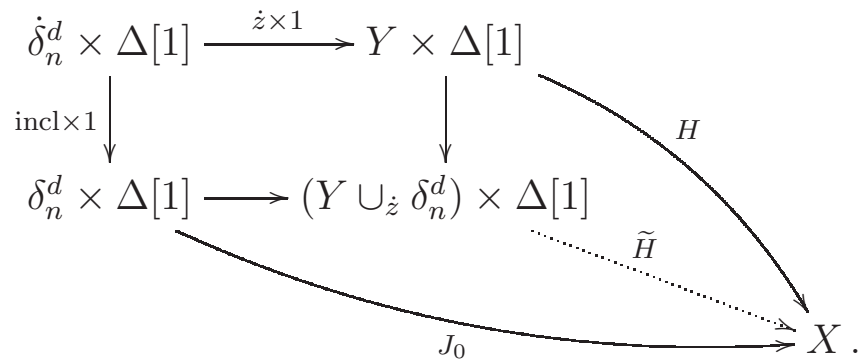

After identifying $Y \cup_{\dot{z}} \delta_{n}^{d}$ with its image $Y \cup\langle z\rangle=\operatorname{Im}\left(\operatorname{incl} \cup_{\dot{z}} z\right) \subseteq X$, the pair $(Y \cup\langle z\rangle, \widetilde{H})$ contradicts the maximality of $(Y, H)$. It follows that $Y=X$.

Step 6. The restriction $q=\left.p\right|_{\widehat{X}}$ is a minimal fibration.

Since $\left.p\right|_{\widehat{X}}$ is a retraction of $p$, it is a fibration. By construction $\widehat{X}$ is a free diagram and if $w$ is a generator of $\widehat{X}$, then it is also a generator of $X$ that belongs to $\Sigma^{\prime}$. Then, none of such simplices can be sub- $p$-homotopic, as otherwise the minimality condition that satisfies $\Sigma^{\prime}$ would be contradicted. Therefore $q$ is a minimal fibration.

Now we will establish a minimality condition for fibrations.

Proposition 3.9. Let $\mathcal{C}$ be an artinian EI-category. Assume that $X$ is a free $\mathcal{C}$-diagram and $p: X \longrightarrow B$ is a fibration in $\mathbf{S}^{\mathcal{C}}$. Then $p$ is minimal if and only if any strong fibrewise deformation retract of $X$ coincides with $X$. 
Proof. Assume that $p: X \longrightarrow B$ is minimal and let $D \leq X$ be a strong fibrewise deformation retract of $X$, and denote by $j$ and $r$ the inclusion and the retraction, respectively. There is a commutative diagram of fibrations:

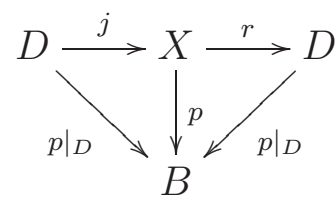

Assume that $D$ and $X$ coincide up to dimension $n-1$, and let $z$ be a simplex of $X$ of dimension $n$, with characteristic map $\delta_{n}^{c} \stackrel{z}{\longrightarrow} X$. Assume furthermore that $z \in \Sigma=\Sigma(X)$ belongs to the generating set of $X$.

Let $H: X \times \Delta[1] \longrightarrow X$ be a fibrewise homotopy relative to $D$ between the identity $\operatorname{Id}_{X}$ and $r \circ j$. Since the boundary $\dot{z}$ of the simplex $z$ belongs to $D$, the composition

$$
\delta_{n}^{c} \times \Delta[1] \stackrel{z \times 1}{\longrightarrow} X \times \Delta[1] \stackrel{H}{\longrightarrow} X
$$

defines a $p$-homotopy $z \simeq_{p} j r(z)$. If $w$ is the generator of the orbit of $j r(z), w \preceq z$. Since both $z$ and $w$ are in $\Sigma$ and $p: X \longrightarrow B$ is minimal, they must coincide. Hence there is an endomorphism $f$ of $\mathcal{C}$ such that with $f(z)=j r(z)$; but as $\mathcal{C}$ is an EI-category, $f$ is an isomorphism, and thus $z=f^{-1}(r(z))$. Since $r(z)$ belongs to $D$, it holds that $z \in D$. So we have proved that every generator of $X$ in dimension $n$ belongs to $D$; and as they coincide in dimension $n-1$, we obtain by induction the desired equality $D=X$.

The other implication is a consequence of Theorem $\mathrm{A}$

The next proposition states a useful technical property of minimal fibrations.

Proposition 3.10. Let $\mathcal{C}$ be a small EI-category, $p: X \longrightarrow B$ a fibration of $\mathcal{C}$-diagrams and $Z$ another $\mathcal{C}$-diagram. Assume that $\alpha, \beta: Z \longrightarrow X$ are fibrewise homotopic maps and $p$ is a minimal fibration. If $\beta$ is an isomorphism, then $\alpha$ is also an isomorphism.

Proof. We will show that $\alpha$ is an isomorphism by induction on the dimension $n$. Notice that for negative dimension $\alpha$ is the isomorphism between empty sets. Hence, we will assume that $\alpha$ is an isomorphism in dimension $k<n$, and will prove that it is also an isomorphism in dimension $n$.

Step 1. The map $\alpha$ is surjective in dimension $n$.

It is sufficient to show that every $n$-simplex of $\Sigma=\Sigma(X)$ is in the image of $\alpha$. Let $x$ be an $n$-simplex of $\Sigma=\Sigma(X)$. By induction hypothesis, $\dot{x} \in \operatorname{Im}(\alpha)$, and then if $x: \delta_{n}^{c} \longrightarrow X$ is the characteristic map for $x$ (that we still denote by the same symbol), there is a map $u: \dot{\delta}_{n}^{c} \longrightarrow Z$ and a commutative diagram in $\mathbf{S}^{\mathcal{C}}$ :

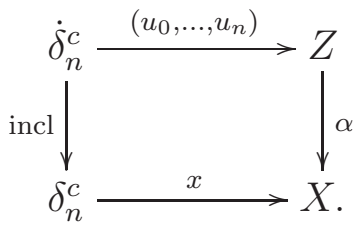


Let $H: Z \times \Delta[1] \longrightarrow X$ be a fibrewise homotopy from $\beta$ to $\alpha$, that is, $H_{0}=\beta$ and $H_{1}=\alpha$. Then, the above diagram extends to a new diagram of solid arrows

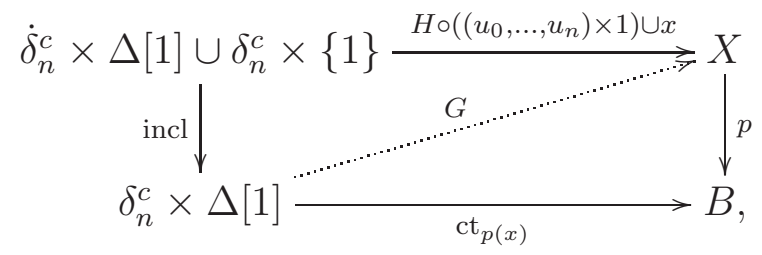

where $\operatorname{ct}_{p(x)}$ is the constant homotopy of the characteristic map of the $\operatorname{simplex} p(x) \in B$ : $\delta_{n}^{c} \stackrel{x}{\longrightarrow} X \stackrel{p}{\longrightarrow} B$. Corollary 2.15 implies that the left side vertical map is a trivial cofibration, and so Axiom M4 of 2.12 implies that there is a homotopy $G: \delta_{n}^{c} \times \Delta[1] \longrightarrow X$ making the whole diagram commutative. By construction $G_{1}$ coincides with the characteristic map of $x$. Then, $w=G_{0}$ determines a new simplex $w \in X$ such that $p(w)=p(x)$ and $w$ and $x$ are homotopic, although not relative to the boundary. In fact, $\left.G\right|_{\dot{\delta}_{n}^{c} \times \Delta[1]}=H \circ\left(\left(u_{0}, \ldots, u_{n}\right) \times 1\right)$ is not necessarily a constant homotopy.

Notice that by construction $\dot{w}=\left(\beta\left(u_{0}\right), \ldots, \beta\left(u_{n}\right)\right)$, and since $\beta$ is an isomorphism, there is a simplex $z \in Z$ such that $\beta(z)=w$, and then $\dot{z}=\left(u_{0}, \ldots, u_{n}\right)$. Now, we want to compose suitably the homotopies $G$ from $w$ to $x$ and $H \circ(z \times 1)$, which is a homotopy from $\beta(z)=w$ to $\alpha(z)$. This is achieved by obtaining again a lifting $F$ :

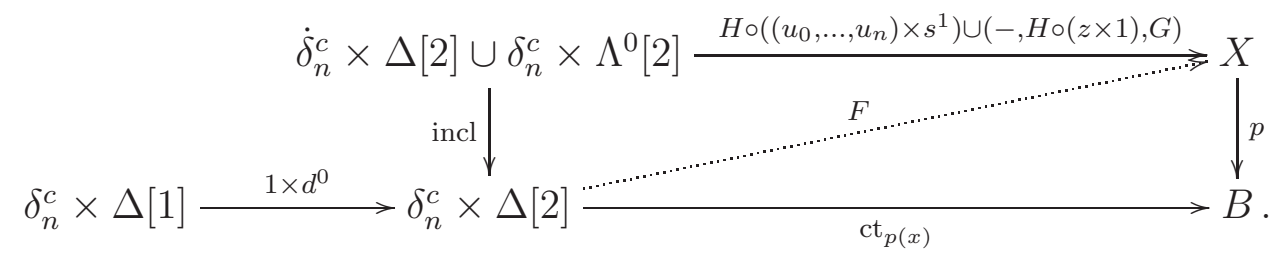

The composite $F \circ\left(1 \times d^{0}\right)$ is now a homotopy from $x$ to $\alpha(z)$. The restriction to the boundary is $\left.F \circ\left(1 \times d^{1}\right)\right|_{\dot{\delta}_{n}^{c} \times \Delta[1]}=H \circ\left(u \times s^{1}\right) \circ\left(1 \times d^{0}\right)=H \circ\left(u \times d^{0} s^{0}\right)$, which is the constant homotopy of $H_{1} \circ u$, the characteristic map of $\alpha(u)$, and then $\dot{x}=\alpha(\dot{z})=\alpha(u)$. Let $y \in \Sigma$ be the generator of the orbit of the simplex $\alpha(z)$ in $X$. We have shown that $y \preceq x$, and hence $y=x$ since both are in $\Sigma$. Since $\mathcal{C}$ is an $E I$-category, there is an isomorphism $f \in \mathcal{C}$ such that $f(x)=\alpha(z)$. Hence, $x=\alpha\left(f^{-1} z\right) \in \operatorname{Im}(\alpha)$.

Step 2. If $z$ and $w$ are two $n$-simplices of $Z$ such that $\alpha(z)=\alpha(w)$, then $\beta(z) \simeq_{p} \beta(w)$.

By the induction hypothesis, $z$ and $w$ must have the same boundary. Since $\alpha(z)=\alpha(w)$, also $\alpha\left(d_{i} z\right)=\alpha\left(d_{i} w\right)$ for all $i=0, \ldots, n$. But $\alpha$ is injective in dimension $n-1$, and thus $d_{i} z=d_{i} w$ for all $i=0, \ldots, n$, that is, $\dot{z}=\dot{w}$.

As in Step 1, let $H$ be a fibrewise homotopy from $\beta$ to $\alpha$. Then, $H \circ(w \times 1)$ is a homotopy from $\beta(w)$ to $\alpha(w)$ (or rather the corresponding characteristic maps). Similarly, $H \circ(z \times 1)$ is a homotopy from $\beta(z)$ to $\alpha(z)$. But $\alpha(w)=\alpha(z)$, so we will combine both homotopies in order to get another one between $\beta(w)$ and $\beta(z)$.

Notice that $H \circ(w \times 1)$ and $H \circ(z \times 1)$ coincide over the boundary $\dot{w}=\dot{z}$, and denote by

$$
h=\left.H \circ(w \times 1)\right|_{\dot{\delta}_{n}^{c} \times \Delta[1]}=\left.H \circ(z \times 1)\right|_{\dot{\delta}_{n}^{c} \times \Delta[1]}
$$


the restriction. We have a commutative diagram of solid arrows

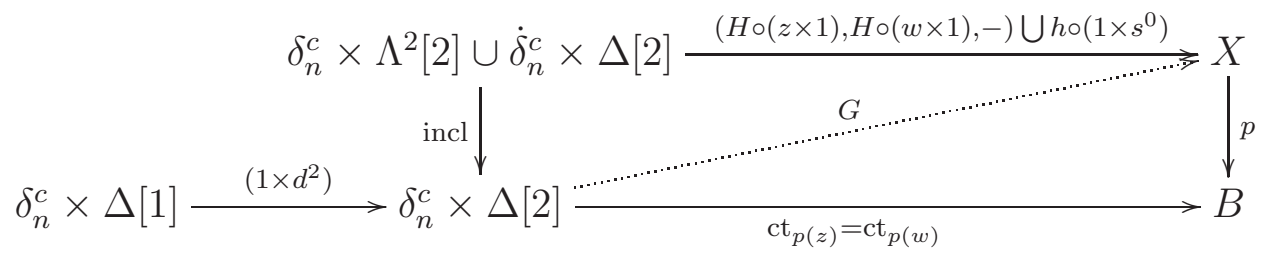

and so, by Corollary 2.15 and the fact that $p: X \rightarrow B$ is a fibration, there is a lift $G$ and the composition $G \circ\left(1 \times d^{2}\right)$ is a $p$-homotopy from $\beta(w)$ to $\beta(z)$.

Step 3. The map $\alpha$ is injective in dimension $n$.

Let $x$ and $y$ be $n$-simplices of $Z$ such that $\alpha(x)=\alpha(y)$. Since $\beta: Z \longrightarrow X$ is an isomorphism, $Z$ is also a free $\mathcal{C}$-diagram with base set $\Sigma(Z)=\beta^{-1}(\Sigma(X))$. Then, there are $x_{0}, y_{0} \in \Sigma(Z)$ and $f, g$ in $\mathcal{C}$ such that

$$
f\left(x_{0}\right)=x \quad \text { and } \quad g\left(y_{0}\right)=y .
$$

Notice that $f\left(\alpha\left(x_{0}\right)\right)=\alpha(x)=\alpha(y)=g\left(\alpha\left(y_{0}\right)\right)$, so there exists $w \in \Sigma(X)$ and morphisms $h, l$ in $\mathcal{C}$ such that

$$
h(w)=\alpha\left(x_{0}\right), l(w)=\alpha\left(y_{0}\right) \text { and } \quad f \circ h=g \circ l .
$$

By Step 1, $\alpha$ is surjective in dimension $n$, so there exists $v \in Z$ such that $\alpha(v)=w$, and thus we have $\alpha(h(v))=h(\alpha(v))=h(w)=\alpha\left(x_{0}\right)$ and $\alpha(l(v))=l(\alpha(v))=l(w)=\alpha\left(y_{0}\right)$. Therefore, we can apply Step 2 in order to conclude that

$$
\beta(h(v)) \simeq_{p} \beta\left(x_{0}\right) \text { and } \beta(l(v)) \simeq_{p} \beta\left(y_{0}\right) .
$$

Now, there is also a simplex $u \in \Sigma(X)$ and a morphism $r$ in $\mathcal{C}$ such that $r(u)=\beta(v)$. Then $h(r(u))=h(\beta(v))=\beta(h(v)) \simeq_{p} g\left(x_{0}\right)$, and also $l(r(u))=l(\beta(v))=\beta(l(v)) \simeq_{p} \beta\left(y_{0}\right)$, so we have shown that

$$
u \preceq \beta\left(x_{0}\right) \text { and } u \preceq \beta\left(y_{0}\right) .
$$

However, $x_{0}, y_{0} \in \Sigma(Z)$, and hence the three simplices $u, \beta\left(x_{0}\right), \beta\left(y_{0}\right)$ are in $\Sigma(X)$. Since $p: X \longrightarrow B$ is minimal, $\beta\left(x_{0}\right)=u=\beta\left(y_{0}\right)$ and $h \circ r=l \circ r$ must be an endomorphism, and then an automorphism of $\mathcal{C}$. Furthermore, $x_{0}=y_{0}$ since $\beta$ is isomorphism. Finally, we have $f \circ(h \circ r)=(f \circ h) \circ r=(g \circ l) \circ r=g \circ(l \circ r)$, thus $f=g$. So we obtain $x=f\left(x_{0}\right)=g\left(y_{0}\right)=y$.

Remark 3.11. It is not true in general that a minimal fibration in $\mathcal{S}^{\mathcal{C}}$ is minimal objectwise, that is, if $p: X \longrightarrow B$ is a minimal fibration of $\mathcal{C}$-diagrams, then it is not always true that for every object $a$ of $\mathcal{C}, p_{a}: X_{a} \longrightarrow B_{a}$ is a minimal fibration in $\mathbf{S}$. The following is an illustrative example. Consider the category $\mathcal{C}=\{a \stackrel{f}{\longrightarrow} b \stackrel{g}{\longleftarrow} c\}$ and a minimal fibration $p: X \longrightarrow B$, where $X$ is a free diagram. In this case $p_{a}: X_{a} \longrightarrow B_{a}$ and $p_{c}: X_{c} \longrightarrow B_{c}$ are minimal, but there might exist generators $z \in X_{a}$ and $w \in X_{c}$ such that $f(z) \neq g(w)$ and $f(z) \simeq_{p_{b}} g(w)$, so $p_{a}: X_{a} \longrightarrow B_{a}$ would not be a minimal fibration of simplicial sets.

Corollary 3.12. Any two minimal fibrations over a diagram B that are fibrewise homotopy equivalent, are isomorphic.

Proof. Let $p: X \rightarrow B$ and $q: Y \rightarrow B$ be minimal fibrations over $B$ and let $\alpha: X \rightarrow Y$ and $\beta: Y \rightarrow X$ be fibrewise homotopy inverses. The composition $\beta \circ \alpha$ is then fibrewise homotopic to the identity map of $X$, and so Proposition 3.10 implies that $\beta \circ \alpha$ is an isomorphism. A similar argument shows that $\alpha \circ \beta$ is also an isomorphism, hence both $\alpha$ and $\beta$ are isomorphisms over $B$. 
We end this section with an example showing how Theorem $\mathrm{A}$ fails on non-artinian index categories.

Example 3.13. Write $\mathcal{N}$ for the poset of non-positive integers $\{\cdots<n<n+1<\cdots<$ $-1<0\}$. A $\mathcal{N}$-diagram $X$ is a sequence of spaces and maps

$$
\cdots \stackrel{f_{n}}{\longrightarrow} X_{n} \stackrel{f_{n+1}}{\longrightarrow} X_{n+1} \stackrel{f_{n+2}}{\longrightarrow} \cdots \stackrel{f_{-1}}{\longrightarrow} X_{-1} \stackrel{f_{0}}{\longrightarrow} X_{0} .
$$

Assume that each $X_{i}$ is non-empty, fibrant and connected, and that each $f_{n}$ is injective. Then

(1) $X$ is a fibrant and it is a free diagram if and only if each simplex has only a finite number of preimages.

(2) $X$ is never minimal and it does not contain a minimal retract.

In any case $X$ is fibrant since each $X_{i}$ is a fibrant simplicial set.

Define $\Sigma$ as the union of the sets $\Sigma_{n}, n \leq 0$, where $\Sigma_{n}$ is the set of simplices of $X_{n}$ that do not belong to the image of $f_{n}: X_{n-1} \longrightarrow X_{n}$. If every simplex has a finite number of preimages in the diagram, then every simplex is the image of an element of $\Sigma$ by a morphism of the index category; as since each $f_{i}$ is injective, $\Sigma$ is a base and $X$ is a free diagram. If for some $n \leq 0$ there is a simplex $x \in X_{n}$ which is in the image of $f_{n} \circ \cdots \circ f_{m}$ for all $m<n$, then $X$ is not free. If it was free with base $\Sigma^{\prime}$, then there would be a generator $y \in \Sigma^{\prime}$ with $f_{n} \circ \cdots \circ f_{r+1}(y)=x$, for $y \in X_{r}$. In this situation we could find a preimage $x^{\prime}$ of $x$ in $X_{r-1}$, and hence there would be another generator $z \in \Sigma^{\prime}, z \in X_{s}$ for some $s>r$ such that $f_{r-1} \circ \cdots \circ f_{s+1}(z)=x^{\prime}$. But, since each $f_{i}$ is injective, we would also have $f_{n} \circ \cdots \circ f_{r} \circ \cdots \circ f_{s+1}(z)=x$ and this would contradict uniqueness of generators mapping to a fixed simplex.

Assuming that $X$ is free, we show that it cannot be minimal. Suppose on the contrary that it was minimal. A base $\Sigma$ must contain 0 -simplices, so for a certain $n$ there exists a 0 -simplex $x$ in $\Sigma_{n}$. But since the precedent $X_{i}, i<n$, are non-empty, there must be another $m<n$ and a 0-simplex $y$ in $\Sigma_{m}$. Since $f_{n} \circ \cdots \circ f_{m+1}(y) \in X_{n}$, and $X_{n}$ is connected, we have $y \preceq x$ and this would imply $x=y$, that contradicts $m<n$.

\section{4. $\mathcal{C}$-TWisted CARTESian PRODUCtS AND $\mathcal{C}$-Fibre BUNDLES}

This section contains the proof of Theorem B. After generalizing the concepts of twisted cartesian product (TCP) and fibre bundle to the category of $\mathcal{C}$-diagrams, we show that the classification of $\mathcal{C}$-fibre bundles reduces to the classification of principal $G$-fibre bundles, always with constant base $\mathcal{C}$-diagram. The theory of minimal fibrations developed in Section 3 applies then to show that the classification of fibrations of $\mathcal{C}$-diagrams over constant base, where $\mathcal{C}$ is an artinian EI-category, reduces to that of $\mathcal{C}$-fibre bundles over $B$.

Recall that a simplicial group $G$ is a functor $G: \Delta^{\text {op }} \longrightarrow$ Groups. It can be seen as a sequence of groups $\left\{G_{n}\right\}_{n \geq 0}$ together with face and degeneracy operators subject to the same relations (11) as simplicial sets. The composition with the underlying functor $\mathcal{U}:$ Groups $\longrightarrow$ Sets gives the underlying simplicial set of a simplicial group.

Definition 4.1. Let $\mathcal{C}$ be a small category. A left action of a simplicial group $G$ on a $\mathcal{C}$-diagram $F$ is a map of $\mathcal{C}$-diagrams

$$
t: G \times F \longrightarrow F
$$

denoted $t(g, x)=g \cdot x$, that satisfies the usual axioms for a group action, namely, for any $n \geq 0, c \in \mathrm{Ob}(\mathcal{C}), g, h \in G_{n}$ and $x \in F_{c, n}, g \cdot(h \cdot x)=(g h) \cdot x$ and $e_{n} \cdot x=x$ if $e_{n}$ denotes the unit of $G_{n}$. 
Given a $\mathcal{C}$-diagram $F$, the simplicial group of automorphisms of $F$ is the subsimplicial set $\operatorname{aut}_{\mathcal{C}}(F)$ of $\operatorname{map}_{\mathcal{C}}(F, F)$ which consists of the commutative diagrams

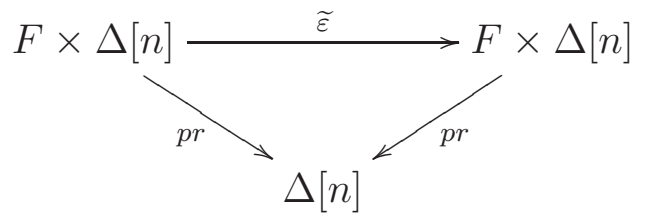

in which the horizontal map is invertible. It clearly becomes a simplicial group that acts on $F$ by evaluation:

$$
\mathrm{ev}: \operatorname{aut}_{\mathcal{C}}(F) \times F \longrightarrow F \text {. }
$$

That is, given $\widetilde{\varepsilon}$ and a simplex $x: \delta_{n}^{c} \longrightarrow F, \operatorname{ev}(\widetilde{\varepsilon}, x)=\varepsilon\left(x, \iota_{n}\right)$, where $\varepsilon$ is the composite of $\widetilde{\varepsilon}$ and the projection $F \times \Delta[n] \longrightarrow F$.

Sometimes we express the action in its adjoint form $\rho: G \longrightarrow \operatorname{aut}_{\mathcal{C}}(F)$. Notice that in that way, an element $g \in G_{n}$ determines an isomorphism of $\mathcal{C}$-diagrams $\rho(g): F \times \Delta[n] \longrightarrow F \times$ $\Delta[n]$ over $\Delta[n]$. Now, given $g \in G_{n}$ and $x \in F_{n}$ with characteristic maps $g: \Delta[n] \longrightarrow G$ and $x: \delta_{n}^{c} \longrightarrow F$, the action $g \cdot x$ is characterized by the composition

$$
\delta_{n}^{c} \stackrel{d}{\longrightarrow} \Delta[n] \times \delta_{n}^{c} \stackrel{g \times x}{\longrightarrow} G \times F \stackrel{\cdot}{\longrightarrow} F .
$$

The classical notion of twisting function will be crucial in the sequel.

Definition $4.2([15, \S 18])$. Let $G$ be a simplicial group and $B$ a simplicial set. Then a twisting function $t: B \longrightarrow G$ is a collection of functions $\left\{t_{n}: B_{n} \longrightarrow G_{n-1}\right\}_{n \geq 1}$ that satisfy:

$$
\begin{aligned}
d_{i} t_{n+1}(v) & =t_{n}\left(d_{i+1} v\right), & & i \geq 1, \\
s_{i} t_{n}(v) & =t_{n+1}\left(s_{i+1} v\right), & & i \geq 0, \\
d_{0} t_{n+1}(v) & =\left[t_{n}\left(d_{0} v\right)\right]^{-1} \cdot t_{n}\left(d_{1} v\right), & & \\
t_{n+1}\left(s_{0} v\right) & =e_{n}, & &
\end{aligned}
$$

for all $n \geq 1$.

Now we have the ingredients we needed in order to define $\mathcal{C}$-twisted cartesian products:

Definition 4.3. Let $G$ be a simplicial group and $F$ a $\mathcal{C}$-diagram with a left action of $G$. Let $B$ be a simplicial set and $t: B \longrightarrow G$ a twisting function, then the $\mathcal{C}$-twisted cartesian product $B \times{ }_{t} F$ is the $\mathcal{C}$-diagram with simplices

$$
\left(B \times_{t} F\right)_{c, n}=B_{n} \times F_{c, n}
$$

and structural operators

$$
\begin{aligned}
d_{0}(b, x) & =\left(d_{0}(b), t(b) \cdot d_{0}(x)\right) & & \\
d_{i}(b, x) & =\left(d_{i} b, d_{i} x\right), & & i \geq 1, \\
s_{i}(b, x) & =\left(s_{i} b, s_{i} x\right), & & i \geq 0, \\
f(b, x) & =(b, f(x)) . & &
\end{aligned}
$$

For short, we will frequently refer to a $\mathcal{C}$-twisted cartesian product simply as a $\mathcal{C}$-TCP.

Once we have extended the concept of twisted cartesian product, we have to deal with fibre bundles. To develop this notion in the context of $\mathcal{C}$-diagrams of spaces we will focus on maps $p: X \longrightarrow B$ of $\mathbf{S}^{\mathcal{C}}$, where $B$ is a constant diagram to the simplicial set $B$. Under this assumption, the classical concepts about local triviality, atlases and structural group (see $[15,11.8]$ ) are generalized, in such a way that we can recover again the theory of $\mathcal{C}$-twisted cartesian products over a constant base space. 
Definition 4.4. Let $F$ be a $\mathcal{C}$-diagram and $B$ a simplicial set. A map $p: X \longrightarrow B$ in $\mathbf{S}^{\mathcal{C}}$, where $B$ is regarded as a constant diagram, will be called a $\mathcal{C}$-fibre bundle with fibre $F$ if $p$ is an epimorphism and for every n-simplex $v \in B$ there exists an isomorphism of $\mathcal{C}$-diagrams, $\alpha_{p}(v): \Delta[n] \times F \longrightarrow \Delta[n] \times{ }_{B} X$, such that the following diagram commutes:

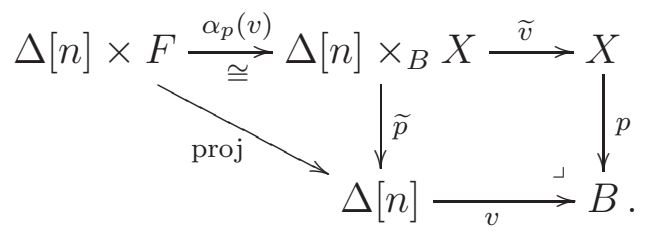

A set of isomorphisms $\left\{\alpha_{p}(v) \mid v \in B_{n}, n \geq 0\right\}$ making commutative the diagram (5) for each simplex $v$ of $B$, is called a $\mathcal{C}$-atlas for $p$.

Set also $\beta_{p}(v)=\widetilde{v} \circ \alpha_{p}(v)$. Notice that the elements $\alpha_{p}(v)$ are isomorphisms over $\Delta[n]$, while $\beta_{p}(v) \in \operatorname{map}_{\mathcal{C}}(F, X)_{n}$ are injections such that the diagram

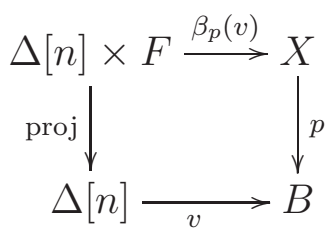

is a pullback diagram of $\mathcal{C}$-diagrams.

Conversely, given a map $\beta_{p}(v)$ making diagram (6) commutative and a pullback diagram, there is a unique factorization $\beta_{p}(v)=\widetilde{v} \circ \alpha_{p}(v)$, where $\alpha_{p}(v): \Delta[n] \times F \longrightarrow \Delta[n] \times_{B} X$ is an isomorphism making (5) commutative, since the right hand square of diagram (5) is a pullback diagram.

It follows that sets of maps $\left\{\alpha_{p}(v)\right\}$ and $\left\{\beta_{p}(v)\right\}$ determine each other. When talking about atlases we will choose $\left\{\alpha_{p}(v)\right\}$ or $\left\{\beta_{p}(v)\right\}$ depending on the context, without further explanation when there is no possible confusion.

Given two atlases $\left\{\alpha_{p}(v)\right\}$ and $\left\{\widetilde{\alpha}_{p}(v)\right\}$ of $p, \alpha_{p}(v)^{-1} \widetilde{\alpha}_{p}(v) \in \operatorname{aut}_{\mathcal{C}}(F)_{n}$, and conversely, if for every $v \in B_{n}$ we choose $(\gamma(v)) \in \operatorname{aut}_{\mathcal{C}}(F)_{n}$, then $\left\{\alpha_{p}(v) \gamma(v)\right\}$ is another atlas.

In the sequel we translate to the context of diagrams some classical notions of Bundle Theory.

Definition 4.5. In the previous notation, the atlas is normal provided $\beta_{p}\left(s_{i} v\right)=s_{i} \beta_{p}(v) \in$ $\operatorname{Hom}_{\mathcal{C}}(F, X)_{n+1}$, for each simplex $v$ of $B_{n}, 0 \leq i \leq n$.

Notice that this equality holds exactly when $\alpha_{p}(v) \circ\left(s^{i} \times 1\right)=\left(\widetilde{s}^{i} \times 1\right) \circ \alpha_{p}\left(s_{i} v\right)$. In other words, the top left square of the following diagram commutes:

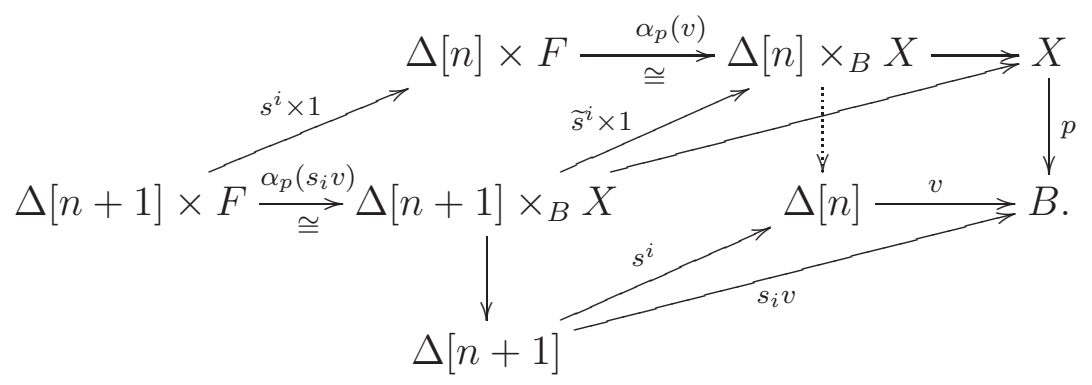


If $\left\{\beta_{p}(v)\right\}$ is an atlas, we can always redefine it over the degenerate simplices using the following definition dimensionwise

$$
\begin{cases}\beta_{p}^{\prime}\left(s_{i} v\right):=s_{i} \beta_{p}(v), & v \in B_{n}, 0 \leq i \leq n, \\ \beta_{p}^{\prime}(v):=\beta_{p}(v), & \text { if } v \text { is non-degenerate }\end{cases}
$$

so it is shown by induction on $n$ that the new atlas $\left\{\beta_{p}^{\prime}(v)\right\}$ is well-defined and normal. Indeed, it is clearly well-defined on 0 -simplices. Assume that it is well-defined on $n$-simplices and that we have two expressions $s_{i} v=s_{j} v^{\prime}$ for a degenerate simplex of dimension $n+1$. It follows that $v^{\prime}=s_{i} d_{j}(v)$ and then $s_{j} \beta_{p}\left(v^{\prime}\right)=s_{i} \beta_{p}(v)$. Thus, upon replacing $\left\{\beta_{p}(v)\right\}$ by $\left\{\beta_{p}^{\prime}(v)\right\}$ we can assume that a given atlas is normal. (cf. [1, pg. 648], [5, 6.5], [15, §19]).

We turn now our attention to face operators. In general $\beta_{p}\left(d_{i} v\right)$ and $d_{i} \beta_{p}(v)$ will differ by an automorphism $\xi_{p}^{i}(v) \in \operatorname{aut}_{\mathcal{C}}(F)_{n-1}, d_{i} \beta_{p}(v)=\beta_{p}\left(d_{i} v\right) \circ \xi_{p}^{i}(v)$, as it is shown in the following commutative diagram:

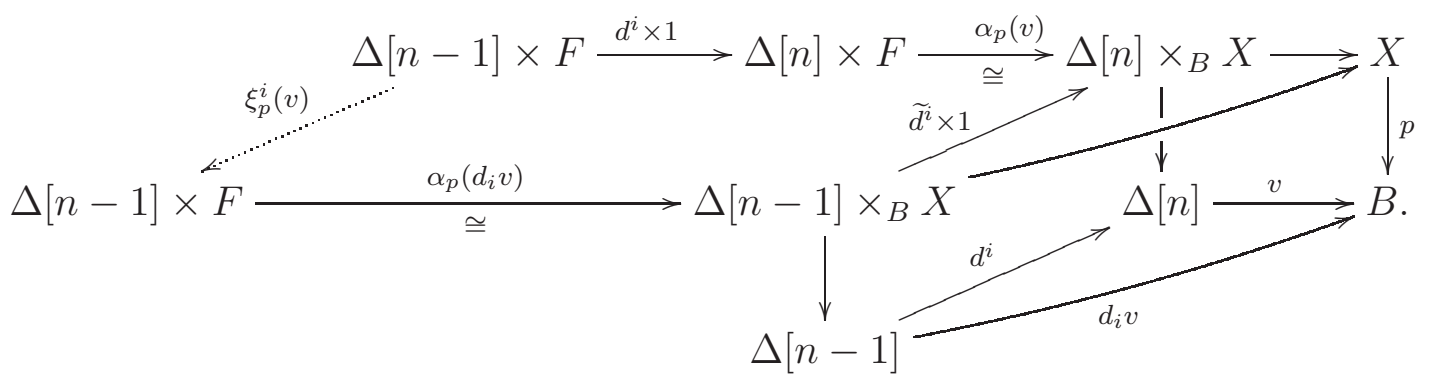

We will refer to $\left\{\xi_{p}^{i}(v) \mid v \in B_{n}, 0 \leq i \leq n\right\}$ as the set of transformation elements associated to the atlas $\left\{\alpha_{p}(v)\right\}$. The following notions generalize classical properties of the atlases:

Definition $4.6\left(\left[1\right.\right.$, IV.2.4], [5, 6.5],[15, 19.1]). (1) An atlas $\left\{\alpha_{p}(v)\right\}$ is regular if for every $v \in B_{n}$ and $1 \leq i \leq n, \xi_{p}^{i}(v)=e_{n-1}$, where $e_{n-1}$ is the identity element of aut $(F)_{n-1}$.

(2) Assume that $G$ is a subgroup complex of $\operatorname{aut}_{\mathcal{C}}(F)$. We will say that $\left\{\alpha_{p}(v)\right\}$ is a $G$-atlas if for each simplex $v$ of $B, \xi_{p}^{i}(v) \in G$.

(3) Two $G$-atlases $\left\{\alpha_{p}(v)\right\}$ and $\left\{\widetilde{\alpha}_{p}(v)\right\}$ are $G$-equivalent if for each $v \in B$, there exists $\gamma(v) \in G$ such that $\widetilde{\alpha}_{p}(v)=\alpha_{p}(v) \gamma(v)$.

Now we can finally define $G$-C $\mathcal{C}$-fibre bundles:

Definition 4.7. A $G$-C-fibre bundle is a $\mathcal{C}$-fibre bundle together with a $G$-equivalence class of $G$-atlases. We call $G$ the structural group of the $\mathcal{C}$-fibre bundle.

Notice that the regularity condition $\xi_{p}^{i}(v)=e_{n-1}$ is equivalent to $\beta_{p}\left(d_{i} v\right)=d_{i} \beta_{p}(v)$, for $i>0$.

In order to work with nice atlases in our $G$-C $\mathcal{C}$-fibre bundles, we need the following statements, that in turn generalize classical results with essentially the same proof.

Proposition 4.8 ([1, 2.5],[5, 6.6],[15, 19.2]). Every equivalence class of $G$-atlases contains a regular $G$-atlas.

Proposition 4.9 ([15, 19.4]). The transformation elements $\left\{\xi_{p}^{0}(v)\right\}$ of a regular $G$-atlas define a twisting function $\xi_{p}^{0}: B \longrightarrow G$.

From now on, given any $G$-C-bundle we may suppose that we are dealing with regular and normalized atlases. The following definition is a generalization of the concept of $G$-map and $G$-equivalence (cf. [15, 19.1]) to the context of $G$-C-fibre bundles. 
Definition 4.10. If $p: X \longrightarrow B$ and $p^{\prime}: X^{\prime} \longrightarrow B$ are $G$-C-fibre bundles with fibre $F$, a $G$ map from $p$ to $p^{\prime}$ is a map of $\mathcal{C}$-diagrams $h: X \longrightarrow X^{\prime}$ such that given $G$-atlases $\left\{\alpha_{p}(v)\right\}$, $\left\{\alpha_{p^{\prime}}(v)\right\}$ of $p$ and $p^{\prime}$, respectively, there exists $\gamma(v) \in G_{n}$ such that $h \beta_{p}(v)=\beta_{p^{\prime}}(v) \gamma(v)$; that is, the following diagram commutes:

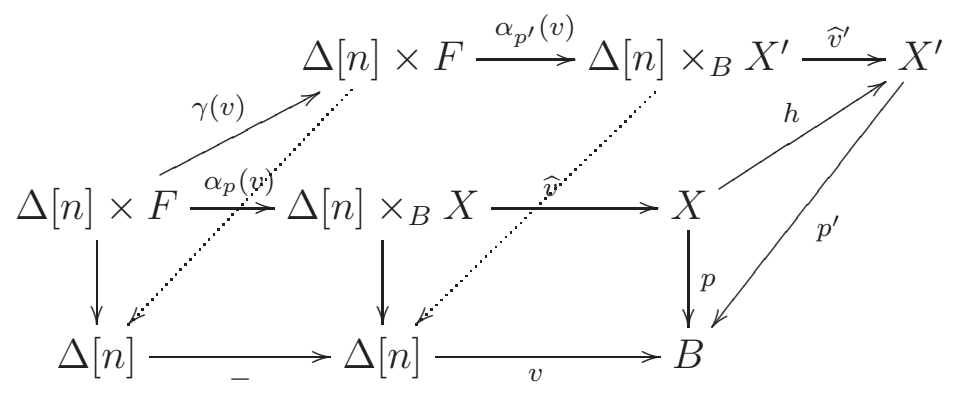

Here we write $\beta_{p}(v)=\widetilde{v} \circ \alpha_{p}(v)$ and $\beta_{p}^{\prime}(v)=\widetilde{v}^{\prime} \circ \alpha_{p}^{\prime}(v)$, as usual.

If $h$ is a natural isomorphism we will say that $p$ and $p^{\prime}$ are G-equivalent. When $G=$ $\operatorname{aut}_{\mathcal{C}}(F)$, an isomorphism $h: X \longrightarrow X^{\prime}$ over $B$ is automatically an $\operatorname{aut}_{\mathcal{C}}(f)$-equivalence. In this case we say that the two $\mathcal{C}$ - fibre bundles are equivalent, with no further mention of the structural group.

Recall (cf. [15, §20]) that two twisting functions $t, t^{\prime}: B \longrightarrow G$ are $G$-equivalent if there is a degree-preserving function $\gamma: B \longrightarrow G$ such that

$$
\begin{aligned}
t^{\prime}(v) \cdot d_{0} \gamma(v) & =\gamma\left(d_{0} v\right) \cdot t(v), & & \\
d_{i} \gamma(v) & =\gamma\left(d_{i} v\right), & & i>0, \\
s_{i} \gamma(v) & =\gamma\left(s_{i} v\right), & & i \geq 0
\end{aligned}
$$

and this is an equivalence relation.

Proposition 4.11. Two G-C-fibre bundles are G-equivalent if and only if the corresponding twisting functions are $G$-equivalent.

Proof. Let $p_{1}: X_{1} \longrightarrow B$ and $p_{2}: X_{2} \longrightarrow B$ be two $G$-C-fibre bundles. Choose $G$-atlases $\left\{\alpha_{1}(v)\right\}$ and $\left(\left\{\beta_{1}(v)\right\}\right)$, and $\left\{\alpha_{2}(v)\right\}$ and $\left(\left\{\beta_{2}(v)\right\}\right)$ for $p_{1}$ and $p_{2}$ respectively.

If $p_{1}$ and $p_{2}$ are $G$-equivalent, then by definition there is a function $\gamma: B \longrightarrow G$ with $h \circ \beta_{2}(v)=\beta_{1}(v) \circ \gamma(v)$, for each simplex $v$ of $B$. Then, if $t_{1}, t_{2}$ denote the respective twisting functions, we have $\beta_{i}\left(d_{0} v\right) \circ t_{i}(v)=d_{0}\left(\beta_{i}(v)\right), i=1,2$. Hence,

$$
\begin{aligned}
\beta_{1}\left(d_{0} v\right) \circ \gamma\left(d_{0} v\right) \circ t_{2}(v) & =h \circ \beta_{2}\left(d_{0} v\right) \circ t_{2}(v) \\
& =h \circ d_{0}\left(\beta_{2}(v)\right) \\
& =d_{0}\left(h \circ \beta_{2}(v)\right) \\
& =d_{0}\left(\beta_{1}(v) \circ \gamma(v)\right) \\
& =d_{0}\left(\beta_{1}(v)\right) \circ d_{0}(\gamma(v))=\beta_{1}\left(d_{0} v\right) \circ t_{1}(v) \circ d_{0}(\gamma(v)),
\end{aligned}
$$

and since $\beta_{1}\left(d_{0} v\right)$ is injective $\gamma\left(d_{0} v\right) \circ t_{2}(v)=t_{1}(v) \circ d_{0}(\gamma(v))$. This is the first condition above (8) and the other two conditions follow similarly. Therefore, $t_{1}$ and $t_{2}$ are $G$-equivalent.

Reading in reverse, the same argument proves that if $t_{1}$ and $t_{2}$ are $G$-equivalent, then $p_{1}$ and $p_{2}$ are $G$-equivalent $G$-C-fibre bundles.

The above notion of $G$-equivalence is translated to the case of $\mathcal{C}$-TCP's as follows. 
Definition 4.12. Two $\mathcal{C}$-TCP's, $B \times \times_{t} F$ and $B \times_{t^{\prime}} F$, with structural group $G$ are $G$ isomorphic if there is a function $\gamma: B \longrightarrow G$ such that $h: B \times_{t} F \longrightarrow B \times_{t^{\prime}} F$ defined by $h(v, x)=(b, \gamma(v) x)$ is an isomorphism of simplicial sets.

The twisting functions codify, in fact, the notion of $G$-isomorphism between $\mathcal{C}$-TCP's:

Proposition 4.13. Two $\mathcal{C}-T C P^{\prime} s B \times{ }_{t} F$ and $B \times \times_{t^{\prime}} F$ with structural group $G$ are isomorphic over $B$ if and only if the twisting functions $t$ and $t^{\prime}$ are equivalent.

Proof. It is easily checked that a function $\gamma: B \longrightarrow G$ satisfies conditions (8), (9), and (10) if and only if $h: B \times_{t} F \longrightarrow B \times \times_{t^{\prime}} F$ defined as $h(v, x)=(v, \gamma(v) x)$ is simplicial.

Proposition 4.14. Let $p: X \longrightarrow B$ be a $\mathcal{C}$-fibre bundle with fibre $F$ and regular $G$-atlas $\left\{\alpha_{p}(v)\right\}$. Then the transformation elements $\left\{\xi_{p}^{0}(v)\right\}$ define a $\mathcal{C}$-twisting map $\xi_{p}^{0}: B_{n} \longrightarrow G_{n-1}$ and thereby $B \times \xi_{p}^{0} F$ becomes a $\mathcal{C}-T C P$ with fibre $F$ and group $G$. Furthermore there is an isomorphism $h: B \times_{\xi_{p}^{0}} F \longrightarrow X$ of $\mathcal{C}$-fibre bundles with group $G$.

Proof. By Proposition 4.9, $\xi_{p}^{0}$ is a twisting function. We can now form the $\mathcal{C}$-TCP $B \times \times_{\xi_{p}^{0}} G$ with a projection $B \times \xi_{p}^{0} G \longrightarrow B$ which is indeed a principal $G$-fibre bundle. Then, we obtain a new $\mathcal{C}$-fibre bundle as $B \times{ }_{\xi_{p}^{0}} F \cong\left(B \times_{\xi_{p}^{0}} G\right) \times_{G} F \longrightarrow B$. We will now construct a $G$-equivalence $h: B \times_{\xi_{p}^{0}} F \longrightarrow X$ over $B$.

For any $n \geq 0, c \in \operatorname{Ob}(\mathcal{C}), v \in B_{n}$, and $z \in F_{c, n}$, define $h_{c}(v, z)=\beta_{p_{c}}(v)\left(\imath_{n}, z\right) \in X_{c, n}$. By Proposition 4.9, $h_{c}$ is an isomorphism for every $c \in \mathcal{C}$. It remains to show that for every $f: c \longrightarrow d \in \mathcal{C}$ the following square commutes:

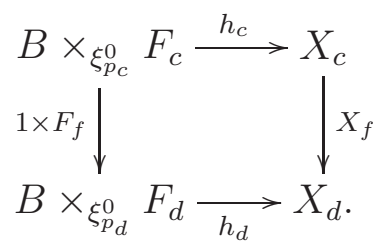

Take an $n$-simplex $(v, z)$ in $B \times_{\xi_{p_{c}}^{0}} F_{c}$ and evaluate it in the above square, that is, $X_{f} h_{c}(v, z)=$ $X_{f} \beta_{p_{c}}(v)\left(\imath_{n}, z\right)$ and $h_{d}\left(1 \times F_{f}\right)(v, z)=h_{d}\left(v, F_{f}(z)\right)=\beta_{p_{d}}(v)\left(\imath_{n}, F_{f}(z)\right)$. Since $p$ is a $\mathcal{C}$-fibre bundle it looks locally as the diagram (7), which is commutative, and therefore $X_{f} \beta_{p_{c}}(v)\left(\imath_{n}, z\right)=$ $\beta_{p_{d}}(v)\left(1 \times F_{f}\right)\left(\imath_{n}, z\right)$, where $\beta_{p_{d}}(v)\left(1 \times F_{f}\right)\left(\imath_{n}, z\right)=\beta_{p_{d}}(v)\left(\imath_{n}, F_{f}(z)\right)$.

Now we can prove the key correspondence between principal $G$-fibre bundles and $G$-C-fibre bundles:

Theorem 4.15. Let $\mathcal{C}$ be a small category, $F$ a $\mathcal{C}$-diagram, and $G$ a simplicial group with a left action on $F$. Given a simplicial set $B$, there is a bijection of sets

$$
\left\{\begin{array}{l}
\begin{array}{l}
\text { Isomorphism classes } \\
\text { of principal } G \text {-fibre } \\
\text { bundles over } B
\end{array}
\end{array}\right\} \stackrel{\Psi}{\longrightarrow}\left\{\begin{array}{l}
G \text {-equivalence classes } \\
\text { of } G \text {-C-fibre bundles } \\
\text { over } B \text { with fibre } F
\end{array}\right\}
$$

which assigns the $G$-class of $G$-C-fibre bundles represented by $p=\xi \times_{G} 1: E \times_{G} F \longrightarrow B$ to the class of a principal $G$-fibre bundle $\xi: E \longrightarrow B$.

Proof. We first check that $\Psi$ is well-defined. A principal $G$-fibre bundle $\xi: E \longrightarrow B$ is locally trivial, hence $p: E \times{ }_{G} F \longrightarrow B$ is also locally trivial, and then a $\mathcal{C}$-fibre bundle. Furthermore, the transformation elements of $\xi$ belong to $G$ and provide a $G$-atlas for $p$, hence $p$ is really a $G$-C-fibre bundle. Finally, if $\xi$ and $\xi^{\prime}$ are isomorphic principal $G$-fibre bundles, they have the same twisting functions by Proposition 4.11, and then Proposition 4.13 implies that $p$ and $p^{\prime}$ are $G$-equivalent. 
We state now the surjectivity of $\Psi$. Given a $G$-C-fibre bundle $p: X \longrightarrow B$, pick a regular $G$ atlas, so the transformation elements form a $G$-twisting function. This function determines a $\mathcal{C}$-TCP $\xi: B \times_{t} G \longrightarrow B$, which is a principal $G$-fibre bundle. Then we should check that $\Psi([\xi])=[p]$, that is, that there is a $G$-equivalence over $B:\left(B \times_{t} G\right) \times_{G} F \stackrel{\cong}{\longrightarrow}$. But this is Proposition 4.14.

Finally, let us check that $\Psi$ is injective. Let $\left[\xi_{1}\right]$ and $\left[\xi_{2}\right]$ be two isomorphism classes of principal $G$-fibre bundles such that $\Psi\left(\left[\xi_{1}\right]\right)=\Psi\left(\left[\xi_{2}\right]\right)$. Fix representatives of these isomorphism classes that are $\mathcal{C}$-TCP's, $\xi_{i}: B \times_{t_{i}} G \longrightarrow B, i=1,2$, with twisting functions $t_{i}: B \longrightarrow G$, $i=1,2$. Then, for $i=1,2, \Psi\left(\left[\xi_{i}\right]\right)$ are represented by the $\mathcal{C}$-TCP's $B \times_{t_{i}} F \cong\left(B \times_{t_{i}} G\right) \times_{G} F$. So, $\Psi\left(\left[\xi_{1}\right]\right)=\Psi\left(\left[\xi_{2}\right]\right)$ if and only if $B \times{ }_{t_{1}} F$ and $B \times{ }_{t_{2}} F$ are $G$-equivalent. By Proposition 4.13 this happens if and only if the twisting functions $t_{1}$ and $t_{2}$ are $G$-equivalent. But Proposition 4.11 implies that this is equivalent to $B \times_{t_{1}} G$ and $B \times_{t_{2}} G$ being isomorphic principal $G$-fibre bundles. In particular, $\left[\xi_{1}\right]=\left[\xi_{2}\right]$.

Proof of Theorem B. This is now an immediate consequence of Theorem 4.15] and the classical classification of isomorphism classes of principal $G$-bundles.

\section{Classification of fibrations}

We proceed now to the proof of Theorem $\mathbb{C}$. The index category $\mathcal{C}$ will be assumed to be a small artinian EI-category.

Lemma 5.1. Let $\mathcal{C}$ be a small category, $\alpha: A \longrightarrow B$ a map in $\mathbf{S}$ and $p: X \longrightarrow B$ a minimal fibration in $\mathbf{S}^{\mathcal{C}}$ over the constant $\mathcal{C}$-diagram to $B$. Then, the pullback fibration $\widetilde{p}: A \times_{B}$ $X \rightarrow A$ is also minimal.

Proof. Let $\Sigma$ be a basis for $X$ in the sense of Definition 2.7. We will show that $\Sigma^{\sqcap}=\{(u, x) \in$ $\left.A \times_{B} X \mid x \in \Sigma\right\}$ is a basis for $A \times_{B} X$. Pick an arbitrary element $(u, x) \in \Sigma$, so $u \in A$ and $x \in \Sigma$. Since $\Sigma$ is closed under degeneracy operators, for each $k, s_{k} x \in \Sigma$ and then also $s_{k}(u, x)=\left(s_{k} u, s_{k} x\right) \in \Sigma^{\sqcap}$, thus $\Sigma^{\sqcap}$ is also closed under degeneracy operators.

Pick now an arbitrary element $(v, z) \in A \times_{B} X$; now $z \in X$ and there is a unique morphism $f$ of $\mathcal{C}$ and a unique $x \in \Sigma$ such that $f(x)=z$. Then, $(v, x) \in A \times_{B} X$ for $p(x)=(p \circ f)(x)=p(z)=\alpha(v)$, so in fact $(v, x) \in \Sigma^{\square}$, and $f(v, x)=(v, z)$. If there were another $g \in \operatorname{Mor}(\mathcal{C})$ and another $(w, y) \in \Sigma^{\sqcap}$ such that $g(w, y)=(v, z)$, we would have that $(w, g(y))=(v, z)=(v, f(x))$, so $w=v$, and since $\Sigma$ is a basis for $X, g=f$ and $y=x$. We have proved that $A \times_{B} X$ is a free $\mathcal{C}$-diagram with base $\Sigma^{\sqcap}$.

In order to prove that the fibration $\widetilde{p}: A \times_{B} X \longrightarrow A$ is minimal it remain to show that given two elements of a given base for $A \times_{B} X$, if one preceds the other then they coincide. Assume that $(v, z)$ and $(w, x)$ are elements of $\Sigma^{\sqcap}$ and suppose that $(w, x) \preceq(v, z)$. Then there exists a map $f \in \mathcal{C}$ such that $f(w, x) \simeq_{p}(v, z)$, so $w=v$ since $p$-homotopic simplices are in the same fibre. We also have that $x \simeq_{p} z$, but from minimality of $p$ it must hold that $x=z$, that is, $(v, z)=(w, x)$.

The proof of the following lemma is analogous to that of Lemma 10.6 in Chapter I of [10]. In the case of $\mathbf{S}^{\mathcal{C}}$ the lifting properties used in this proof can be obtained using Proposition 2.14 and Axiom M4 (see Definition 2.11). This result will help to establish a connection between the theory of minimal fibrations and the theory of $\mathcal{C}$-fibre bundles.

Lemma 5.2. Let $\mathcal{C}$ be a small category, $\alpha_{0}, \alpha_{1}: A \longrightarrow B$ homotopic maps in $\mathbf{S}^{\mathcal{C}}$ and let $p: X \longrightarrow B$ be a fibration in $\mathbf{S}^{\mathcal{C}}$. Then the induced fibrations $p_{\alpha_{0}}: A \times_{B}^{\alpha_{0}} X \longrightarrow A$ and $p_{\alpha_{1}}: A \times_{B}^{\alpha_{1}} X \longrightarrow A$ are fibrewise homotopy equivalent. 
Definition 5.3. The fibre $F_{b}$ of a $\mathcal{C}$-fibration $p: X \longrightarrow B$ over a vertex $b \in B$ is defined by the pullback diagram

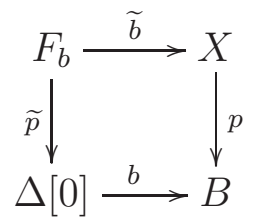

We will say that the fibre of the fibration $p: X \rightarrow B$ is weakly equivalent to the $\mathcal{C}$-diagram $F$ if for every vertex $b \in B$, the fibre $F_{b}$ over $b$ is weakly homotopy equivalent to $F$.

Definition 5.4. Two fibrations $p: X \longrightarrow B$ and $p^{\prime}: X^{\prime} \longrightarrow B$ in $\mathbf{S}^{\mathcal{C}}$ are said to be weakly homotopy equivalent if there exists a finite zig-zag

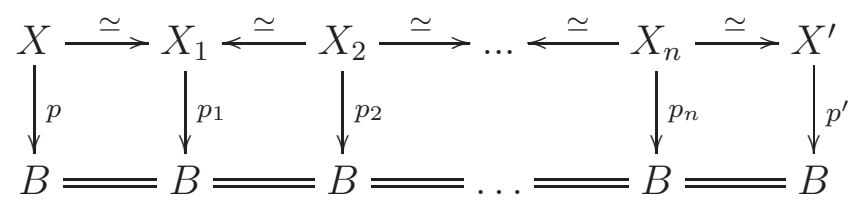

of weak equivalences over $B$ that joins $p$ with $p^{\prime}$ through fibrations of $\mathbf{S}^{\mathcal{C}}$.

In the situation of the above definition, for a vertex $b$ of $B$, we obtain a corresponding zig-zag of weak homotopy equivalences between the respective fibres

$$
F_{b} \stackrel{\simeq}{\longrightarrow}\left(F_{1}\right)_{b} \stackrel{\simeq}{\longleftarrow}\left(F_{2}\right)_{b} \stackrel{\simeq}{\longrightarrow} \ldots \stackrel{\simeq}{\longleftarrow}\left(F_{n}\right)_{b} \stackrel{\simeq}{\longrightarrow} F_{b}^{\prime}
$$

Remark 5.5. Note that we would obtain an equivalent definition to 5.4 if we did not require the maps $p_{i}: X_{i} \longrightarrow B, i=1, \ldots, n$ to be fibrations. Indeed, if $p_{n}$ is not a fibration, we can factor it as a trivial cofibration followed by a fibration $X_{n} \stackrel{\imath}{\longrightarrow} X_{n}^{\prime} \stackrel{p_{n}^{\prime}}{\longrightarrow} B$, by axioms M5 (cf. Definition 2.12) and then axiom M4 would provide liftings $X_{n-1} \stackrel{\simeq}{\longleftarrow} X_{n}^{\prime} \stackrel{\simeq}{\longrightarrow} X^{\prime}$, making the diagram

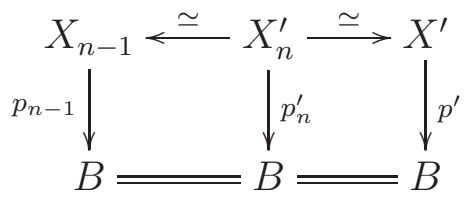

commutative, with $p_{n}^{\prime}$ a fibration. Similarly for the other maps $p_{i}$.

Lemma 5.6. If $p: X \longrightarrow B$ is a minimal $\mathcal{C}$-fibration, the fibre $F_{b}$ over a vertex $b \in B$ is $a$ minimal $\mathcal{C}$-diagram. Moreover,

(a) If $b^{\prime}$ is another vertex of $B$ in the same connected component as $b$, then $F_{b}$ and $F_{b^{\prime}}$ are isomorphic.

(b) If the fibrations in the sequence of weak equivalences (11) are minimal, then the maps in the sequence (12) are isomorphisms.

Proof. $F_{b}$ is minimal by Lemma 5.1 .

Then, (a) follows because if $b$ and $b^{\prime}$ are the two vertices of an edge of $B$, Lemma 5.2 implies that the pullback of $p$ along the inclusion of this edge in $B$ provides a homotopy equivalence between $F_{b}$ and $F_{b^{\prime}}$. Now, Corollary 3.12 implies that the map is indeed an isomorphism. In general there will be a sequence of edges joining $b$ and $b^{\prime}$, and we only need to apply this same argument repeatedly along these lines.

Finally, (b) follows by similar arguments. 
Corollary 5.7. Let $B$ be a connected simplicial set. If $p: X \longrightarrow B$ is a minimal fibration in $\mathbf{S}^{\mathcal{C}}$ over the constant diagram $B$, then $p$ is a $\mathcal{C}$-fibre bundle over $B$ with fibre $F \cong F_{b}$ for any vertex $b \in B$.

Proof. Since $\Delta[n]$ is contractible, the result follows from Lemma 5.1. Corollary 3.12 and Lemma 5.2 .

Definition 5.8. A minimal cofibrant-fibrant replacement for $F$ is a $\mathcal{C}$-diagram $M F$, weakly equivalent to $F$, which is a minimal $\mathcal{C}$-diagram in the sense of Definition 3.3 .

Proposition 5.9. Let $\mathcal{C}$ be a small artinian EI-category.

(a) Each $\mathcal{C}$-diagram $F$ admits a minimal cofibrant-fibrant replacement. If $F$ is a fibrant $\mathcal{C}$-diagram, there is a choice of a minimal cofibrant-fibrant replacement $M F$ for which there is a weak equivalence

$$
\kappa: M F \stackrel{\simeq w}{\longrightarrow} F .
$$

(b) If $B$ is a connected simplicial set, any fibration of $\mathcal{C}$-diagrams $p: X \longrightarrow B$ over the constant diagram $B$ with fibre weakly homotopy equivalent to a $\mathcal{C}$-diagram $F$ is weakly homotopy equivalent to a minimal fibration $\widehat{p}: M X \rightarrow B$ with fibres isomorphic to $M F$, a minimal cofibrant-fibrant replacement of $F$.

Proof. (b) We build a diagram of fibrations

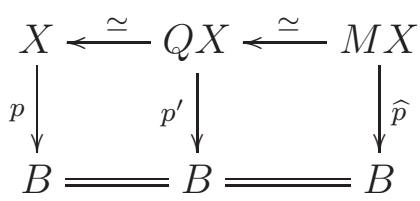

by first applying Quillen's small objects argument to the $\mathcal{C}$-diagram $X$, thus obtaining a trivial fibration $Q X \rightarrow X$ with $Q X$ a free $\mathcal{C}$-diagram (see Remark 2.13). The map $p^{\prime}: Q X \longrightarrow B$, making the square in the left of the above diagram commutative, is then defined as the composition of two fibrations and it is therefore a fibration. By Theorem $\mathrm{A}$, $p^{\prime}: Q X \longrightarrow B$ has a fibrewise deformation retract $\widehat{p}: M X \longrightarrow B$ which is a minimal fibration.

By Lemma 5.6(a) the fibres over the different vertices of $B$ are isomorphic. We can set $M F=(M X)_{b}$, the fibre of $\widehat{p}$ over a chosen vertex $b \in B$.

By pulling back the fibrations of (15) along the inclusion of the vertex $b$, we get a map between the fibres of $p$ and of $\widehat{p}$ which is a weak homotopy equivalence $X_{b} \longleftarrow(M X)_{b}$. By assumption $X_{b} \simeq_{w} F$ are weak homotopy equivalent. $M F=(M X)_{b}$ is a minimal cofibrantfibrant $\mathcal{C}$-diagram, hence a minimal cofibrant-fibrant replacement for $F$.

(a) This follows now from (b) with $B=*$ a point and $X=F$. If $F$ is a fibrant $\mathcal{C}$-diagram, then (15) provides the map $\kappa: M F \stackrel{\simeq_{w}}{\longrightarrow} F$.

If $F$ is not fibrant, then we take first a fibrant approximation $F \longrightarrow \widehat{F}$, and then apply the above construction to $\widehat{F}$ and get $F \stackrel{\simeq_{w}}{\longrightarrow} \widehat{F} \stackrel{\simeq_{w}}{\longleftarrow} M \widehat{F}$.

Proposition 5.10. Let $\mathcal{C}$ be a small artinian EI-category.

(a) Two minimal cofibrant-fibrant replacements for a $\mathcal{C}$-diagram $F$ are isomorphic.

(b) Two weakly homotopy equivalent minimal fibrations of $\mathcal{C}$-diagrams over a constant diagram $B$ are isomorphic.

Proof. (b) Assume that $p: M \longrightarrow B$ and $p^{\prime}: M^{\prime} \longrightarrow B$ are minimal fibrations of $\mathcal{C}$-diagrams. If they are weakly homotopy equivalent, then there is a finite zig-zag of weak equivalences 
of fibrations

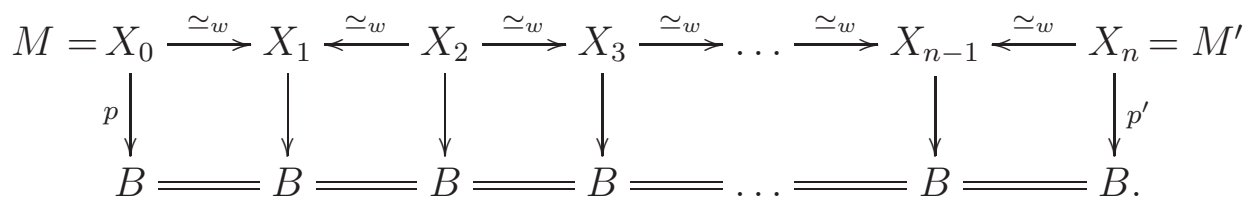

Upon replacing $X_{i}$ by $Q X_{i}, i=1, \ldots, n-1$ (see Remark 2.13) we can assume that all of the $X_{i}$ 's are cofibrant $\mathcal{C}$-diagrams. $M$, and $M^{\prime}$ are also cofibrant since the fibrations $p, p^{\prime}$ are minimal.

Whitehead theorem for model categories implies that these are indeed homotopy equivalences. We will only sketch some small changes to the classical proof (cf. [10, 12]) that in our situation avoids the model category structure of $\mathbf{S}^{\mathcal{C}} \downarrow B$.

We can assume that each of the maps in the top horizontal line of digram (16) is either a trivial cofibration or a trivial fibration. Otherwise, factor a weak equivalence $X_{i} \longrightarrow X_{i \pm 1}$ into a cofibration followed by a trivial fibration $X_{i} \longrightarrow Z \longrightarrow X_{i \pm 1}$ over $B$. It turns out that $X_{i} \longrightarrow Z$ is actually a trivial cofibration and $Z \longrightarrow B$ is a fibration with $Z$ a cofibrant $\mathcal{C}$-diagram.

Assume that one of the maps $f: X_{i} \longrightarrow X_{i \pm 1}$ is a trivial cofibration. In this case, we obtain a left inverse $g$ of $f$ over $B$ as a dotted lift in the following solid diagram

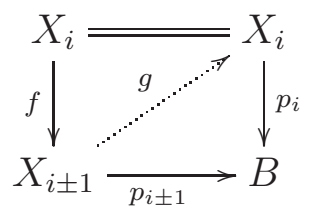

A homotopy $h$ over $B$ between $g \circ f$ and $\operatorname{Id}_{X_{i}}$ is provided by the dotted lift in the following solid diagram

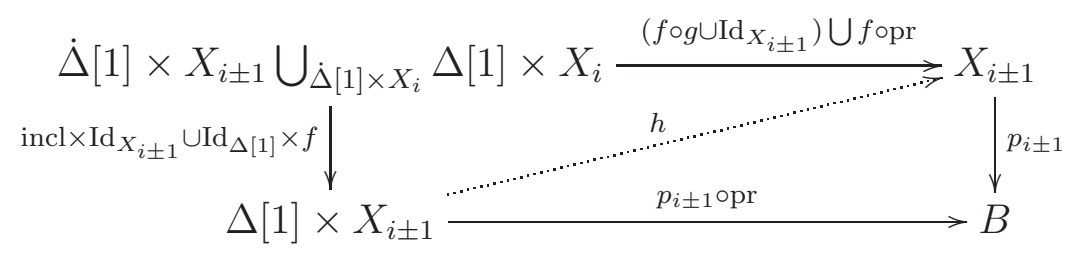

where the left vertical map is a trivial cofibration by Proposition 2.14.

A similar argument shows that if $f: X_{i} \longrightarrow X_{ \pm 1}$ is a trivial fibration, then it is a homotopy equivalence over $B$. Since each of the maps $p_{i}: X_{i} \longrightarrow B$ is a fibration, the composition of the fibrewise homotopy equivalences of diagram (16) is a fibrewise homotopy equivalence $M \longrightarrow M^{\prime}$ over $B$. Now, the result follows by Corollary 3.12 ,

(a) This follows now from (b), taking $B=*$ and $M=F$.

By Corollary [5.7, and assuming that $B$ is connected, a minimal fibration $\widehat{p}: M X \longrightarrow B$ with fibres isomorphic to $M F$, like the one obtained in Proposition 5.9(b), is indeed a a fibre bundle with fibre $M F$ and structural group $\operatorname{aut}_{\mathcal{C}}(M F)$, by default. We will describe the homotopy type of $\operatorname{aut}_{\mathcal{C}}(M F)$ in terms of the original $F$, assuming that $F$ is fibrant. This is based on work of Dwyer and Kan on function complexes [6]. We will need to introduce now the notion of twisted arrow category.

Definition 5.11. Let $\mathcal{C}$ be a small category. The twisted arrow category a $\mathcal{C}$ of $\mathcal{C}$ is the category whose objects are the morphisms $f: a \longrightarrow b$ of $\mathcal{C}$, and morphisms from $f: a \longrightarrow b$ 
to $f^{\prime}: a^{\prime} \longrightarrow b^{\prime}$ the pairs of morphisms $(\alpha, \beta)$, where $\alpha \in \operatorname{Mor}_{\mathcal{C}}\left(a^{\prime}, a\right), \beta \in \operatorname{Mor}_{\mathcal{C}}\left(b, b^{\prime}\right)$, and the diagram

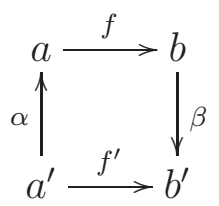

commutes in $\mathcal{C}$.

Following [6], if $X, Y$ are $\mathcal{C}$-diagrams, we define a diagram of function complexes, indexed by the twisted arrow category $a \mathcal{C}$,

$$
\operatorname{map}_{\mathrm{a}}(X, Y): \mathrm{aC} \longrightarrow \mathrm{S}
$$

that maps an object $f: a \longrightarrow b$ to $\operatorname{map}(X(a), Y(b))$ and a morphism $(\alpha, \beta)$ from $f$ to $f^{\prime}$ to the $\operatorname{map} \alpha^{\sharp} \beta_{\sharp}: \operatorname{map}(X(a), Y(b)) \longrightarrow \operatorname{map}\left(X\left(a^{\prime}\right), Y\left(b^{\prime}\right)\right)$ induced by right and left composition.

Naturality of these function complex diagrams is easily checked. A map of $\mathcal{C}$-diagrams $\theta: X^{\prime} \longrightarrow X$ induce a map of $a \mathcal{C}$-diagrams $\theta^{*}: \operatorname{map}_{a}(X, Y) \longrightarrow \operatorname{map}_{a}\left(X^{\prime}, Y\right)$, which for each $\varphi \in \operatorname{map}(X(a), Y(b))$ is defined over every object $f: a \longrightarrow b$ of aC , by the precomposition $\theta_{f}^{*}(\varphi)=\varphi \circ \theta(a)$. Similarly, a map of $\mathcal{C}$-diagrams $\theta: Y \longrightarrow Y^{\prime}$ induce a map of a $\mathcal{C}$-diagrams $\theta_{*}: \operatorname{map}_{\mathrm{a}}(X, Y) \longrightarrow \operatorname{map}_{\mathrm{a}}\left(X, Y^{\prime}\right)$, by postcomposition. It follows that the function complex diagram is a bifunctor

$$
\operatorname{map}_{\mathrm{a}}(-,-):\left(\mathbf{S}^{\mathcal{C}}\right)^{\mathrm{op}} \times \mathbf{S}^{\mathcal{C}} \longrightarrow \mathbf{S}^{\mathrm{aC}}
$$

One clearly has an isomorphism $\operatorname{map}_{\mathcal{C}}(X, Y) \cong \lim _{\mathrm{a} \mathcal{C}} \operatorname{map}_{\mathrm{a}}(X, Y)$ and a natural map

$$
\lim _{\mathrm{a} \mathcal{C}} \operatorname{map}_{\mathrm{a}}(X, Y) \stackrel{\simeq}{\longrightarrow} \underset{\mathrm{a} \mathcal{C}}{\operatorname{holim}} \operatorname{map}_{\mathrm{a}}(X, Y)
$$

which according to [․ Theorem 3.3] is a weak homotopy equivalence. Recall that the inverse limit $\lim _{\mathfrak{a}} \operatorname{map}_{\mathrm{a}}(X, Y)$ can be defined as the function complex map $\operatorname{coc}_{\mathrm{c}}\left(*, \operatorname{map}_{\mathrm{a}}(X, Y)\right)$, where * stands for a constant functor with value a single point. Likewise,

$$
\underbrace{\operatorname{holim}}_{\mathrm{a} \mathcal{C}} \operatorname{map}_{\mathrm{a}}(X, Y)=\operatorname{map}_{\mathrm{a} \mathcal{C}}\left(E(\mathrm{aC}), \operatorname{map}_{\mathrm{a}}(X, Y)\right)
$$

where $E(\mathbf{a C})$ is the cofibrant replacement of $*$ in the model category $\mathbf{S}^{\mathrm{aC}}$, which for each object $f$ of $a \mathcal{C}, E(a \mathcal{C})(f)$ is defined as the nerve of the overcategory aC $\downarrow f . E(a \mathcal{C})$ is indeed a free $\mathbf{a} \mathcal{C}$-diagram. The map in $(18)$ is then induced by the natural projection $E(\mathrm{aC}) \longrightarrow *$.

Lemma 5.12. Let $\mathcal{C}$ be a small category and $X$ and $Y \mathcal{C}$-diagrams. Assume that $Y$ is fibrant, then

(a) $\operatorname{map}_{\mathrm{a}}(X, Y)$ is a fibrant aC-diagram, and

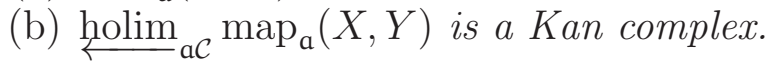

Proof. Since $Y$ is fibrant, for each $c \in \mathcal{C}, Y(c)$ is a Kan complex. Then, for each object $f: a \longrightarrow b$ in the arrow category, $\operatorname{map}_{a}(X, Y)(f)=\operatorname{map}(X(a), Y(b))$ is also a Kan complex (cf. [15, I.6.9]), so $\operatorname{map}_{a}(X, Y)$ is a fibrant aC-diagram. Using the description of the homotopy inverse limit in equation (19), (b) follows essentially from axiom M7 in Definition 2.12 (see also [10, II.3.2], [12, 9.3.1]), since $E(a \mathcal{C})$ is cofibrant and $\operatorname{map}_{a}(X, Y)$ is fibrant.

For each object $f: a \longrightarrow b$ of $a \mathcal{C}, E(a \mathcal{C})(f)$ has a canonical base point, the vertex $v_{f}$ corresponding to the terminal object of the overcategory $a \mathcal{C} \downarrow f$. Evaluating at the vertices 
$v_{\mathrm{Id}_{a}}, a \in \mathrm{Ob}(\mathcal{C})$, we obtain a map

$$
\underset{\mathrm{aC}}{\operatorname{holim}} \operatorname{map}_{\mathrm{a}}(X, Y) \stackrel{\text { ev Id }}{\longrightarrow} \prod_{a \in \mathrm{aC}} \operatorname{map}(X(a), Y(a))
$$

such that the composition

$$
\operatorname{map}_{\mathcal{C}}(X, Y) \cong \underbrace{\lim }_{\mathrm{a} \mathcal{C}} \operatorname{map}_{\mathrm{a}}(X, Y) \longrightarrow \underset{\mathrm{aC}}{\operatorname{holim}} \operatorname{map}_{\mathfrak{a}}(X, Y) \rightarrow \prod_{a \in \mathfrak{a} \mathcal{C}} \operatorname{map}(X(a), Y(a))
$$

maps a natural transformation $\eta: X \longrightarrow Y$ to the sequence $\left\{\eta_{a}: X(a) \longrightarrow Y(a)\right\}_{a \in \mathrm{Ob}(\mathrm{aC})}$ of the natural maps defined for the different objects of $\mathcal{C}$. This will help to identify the connected components of holim ${ }_{\mathrm{aC}} \operatorname{map}_{\mathrm{a}}(X, Y)$ that correspond to invertible maps in $\operatorname{map}_{\mathcal{C}}(X, Y)$, as stated in the following definition.

Definition 5.13. Let $X$ be a fibrant $\mathcal{C}$-diagram. We define the space haut ${ }_{\mathcal{C}}(X)$ of self homotopy equivalences of $X$ as the subspace $\left[\operatorname{holim}_{a \mathcal{C}} \operatorname{map}_{a}(X, X)\right]_{\text {we }}$ of holim $\operatorname{map}_{a}(X, X)$ consisting of the connected components of the vertices $\omega$ of ${\underset{\leftarrow}{\swarrow} \lim _{\mathrm{c}}}_{\operatorname{map}_{\mathrm{a}}(X, Y) \text { such that }}$ the evaluations $\operatorname{ev}_{\text {Id }}(\omega)=\left\{\omega_{a}: X(a) \longrightarrow Y(a)\right\}_{\mathrm{a} \in \mathrm{Ob}(\mathcal{C})}$ are weak equivalences. In case $X$ is not fibrant, we define $\operatorname{haut}_{\mathcal{C}}(X) \stackrel{\operatorname{def}^{\prime}}{=} \operatorname{haut}_{\mathcal{C}}(\widehat{X})$, where $\widehat{X}$ is a fibrant replacement of $X$.

Proposition 5.14. If $X$ is a fibrant $\mathcal{C}$-diagram, then haut ${ }_{\mathcal{C}}(X)$ is a loop space with classifying space $B$ haut $_{\mathcal{C}}(X) \simeq \bar{W} \operatorname{aut}_{\mathcal{C}}(M X)$, where $M X$ is a minimal cofibrant-fibrant replacement for $X$.

Proof. Notice first that according to Proposition 5.10(a), the conclusion of the proposition does not depend on the choice of $M X$. Furthermore, we can fix a model for $M X$ together with weak homotopy equivalence $\kappa: M X \stackrel{\simeq_{w}}{\longrightarrow} X$ (cf. Proposition [5.9)(a)).

We need to prove that the space of loops $\Omega\left(\bar{W} \operatorname{aut}_{\mathcal{C}}(M X)\right)$ is homotopy equivalent to $\operatorname{haut}_{\mathcal{C}}(X)$, so it will be enough to show a homotopy equivalence $\operatorname{aut}_{\mathcal{C}}(M X) \simeq \operatorname{haut}_{\mathcal{C}}(X)$.

Observe that by [6, Theorem 3.3], there is a sequence of homotopy equivalences

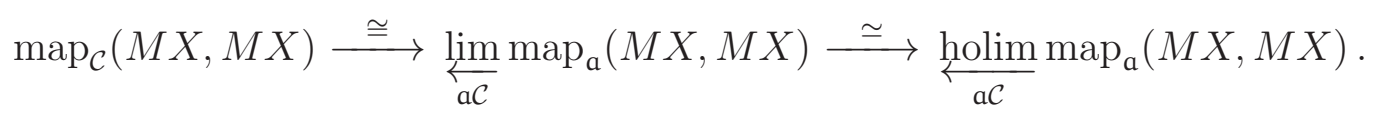

Since $\kappa: M X \longrightarrow X$ is a weak equivalence of $\mathcal{C}$-diagrams, for each object $c$ of $\mathcal{C}$, the map $\kappa_{c}:(M X)(c) \longrightarrow X(c)$ is a weak equivalence of simplicial sets. By naturality of map (17), $\kappa$ induces maps of aC-diagrams

$$
\operatorname{map}_{\mathrm{a}}(X, X) \stackrel{\kappa^{*}}{\longrightarrow} \operatorname{map}_{\mathrm{a}}(M X, X) \stackrel{\kappa_{*}}{\longleftarrow} \operatorname{map}_{\mathrm{a}}(M X, M X)
$$

By assumption $X$ is fibrant and so is $M X$, hence by Lemma 5.12, all three aC-diagrams in the above equation (23) are fibrant. Furthermore the maps $\kappa^{*}$ and $\kappa_{*}$ are weak equivalences in $\mathbf{S}^{\mathrm{aC}}$. In fact, for each object $f: a \longrightarrow b$ of $a \mathcal{C}$, the natural map

$$
\kappa_{f}^{*} \stackrel{\text { def }}{=} \kappa_{a}^{\sharp}: \operatorname{map}(X(a), X(b)) \longrightarrow \operatorname{map}((M X)(a), X(b)),
$$

is given by precomposition with the map $\kappa_{a}:(M X)(a) \longrightarrow X(a)$ which is a weak equivalence of simplicial sets and $X(b)$ is a Kan complex; therefore we obtain that $\kappa_{f}^{*}=\kappa_{a}^{\sharp}$ is a weak equivalence (cf. [15, I.6.9]). A similar argument applies to $\kappa_{*}$.

Now, Bousfield-Kan homotopy lemma [4, XI.5.6] applies to show that the induced maps

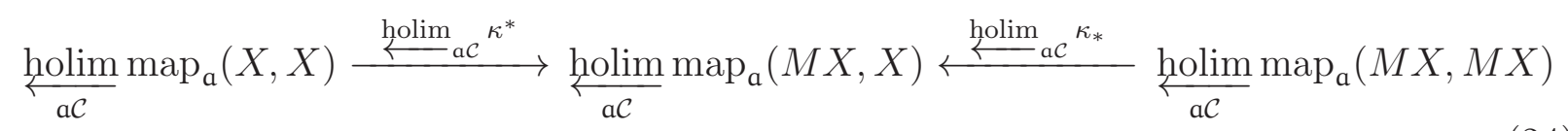


are weak homotopy equivalences. In other words, this follows from [12, 9.3.3(1)], by our description of homotopy inverse limit in Equation (19) and because the diagram $E(a \mathcal{C})$ is cofibrant in the simplicial model category of $\mathrm{a} \mathcal{C}$-diagrams.

The weak equivalences of equations (22) and (24) combine to provide the following zig-zag diagram

$$
\operatorname{map}_{\mathcal{C}}(M X, M X) \longrightarrow \underset{\mathrm{a} \mathcal{C}}{\operatorname{holim}} \operatorname{map}_{\mathrm{a}}(M X, X) \longleftarrow \underset{\mathrm{aC}}{\operatorname{holim}} \operatorname{map}_{\mathrm{a}}(X, X) .
$$

Since the above maps are weak equivalences between Kan complexes, they are also homotopy equivalences (cf. [10, II.1.10]) and we we have already obtained a homotopy equivalence $\operatorname{map}_{\mathcal{C}}(M X, M X) \simeq \operatorname{holim}_{\mathrm{aC}} \operatorname{map}_{\mathrm{a}}(X, X)$. It only remains to show that the connected components of $\operatorname{map}_{\mathcal{C}}(M X, M X)$ that consist of isomorphisms correspond to those of $\operatorname{haut}_{\mathcal{C}}(X)$

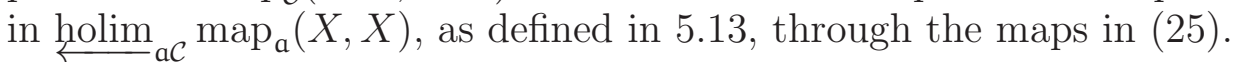

Choose a vertex $\eta \in \operatorname{aut}_{\mathcal{C}}(M X) \subseteq \operatorname{map}_{\mathcal{C}}(M X, M X)$, that is, a natural isomorphism $\eta: M X \longrightarrow M X$ of $\mathcal{C}$-diagrams. The image of $\eta$ along the left map in diagram (25), will be a vertex $\widetilde{\eta} \in \operatorname{holim}_{\mathrm{aC}} \operatorname{map}_{\mathrm{a}}(M X, X)$ such that $\operatorname{ev}_{\mathrm{Id}}(\widetilde{\eta})=\left\{\widetilde{\eta}_{a}:(M X)(a) \longrightarrow X(a)\right\}_{\mathrm{a} \in \mathrm{Ob}(\mathcal{C})}$ where $\widetilde{\eta}_{a}$ is the composition

$$
M X(a) \stackrel{\eta_{a}}{\longrightarrow} M X(a) \stackrel{f_{a}}{\longrightarrow} X(a),
$$

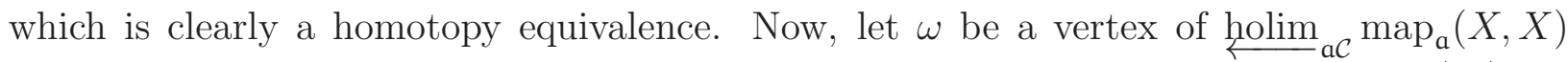
mapping to the same connected component as $\widetilde{\eta}$ along the right map of diagram (25). It follows that if $\operatorname{ev}_{\mathrm{Id}}(\sigma)=\left\{\omega_{a}: X(a) \longrightarrow X(a)\right\}_{\mathrm{a} \in \mathrm{Ob}(\mathcal{C})}$, there is a diagram

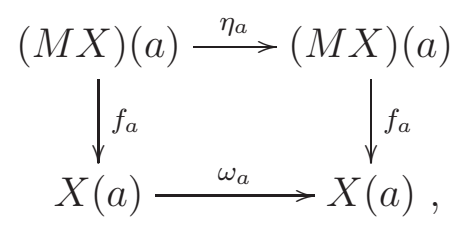

for each object $a \in \mathcal{C}$, that commute up to homotopy. It follows that each $\omega_{a}$ is a weak homotopy equivalence, and $\omega$ is a vertex of $\operatorname{haut}_{\mathcal{C}}(X)$ by Definition 5.13.

Conversely, choose a 0 -simplex $\omega$ of $\operatorname{haut}_{\mathcal{C}}(X) \subseteq \operatorname{holim}_{\mathrm{aC}} \operatorname{map}_{\mathrm{a}}(X, X)$ and write $\mathrm{ev}_{\mathrm{Id}}(\omega)=$ $\left\{\omega_{a}: X(a) \longrightarrow X(a)\right\}_{\mathrm{a} \in \mathrm{Ob}(\mathcal{C})}$, where each $\omega_{a}$ is a weak homotopy equivalence. Observe that $\omega$ determines a connected component in holim ${ }_{a c} \operatorname{map}_{a}(X, X)$. Choose a vertex $\eta$ in the corresponding connected component of $\operatorname{map}_{\mathcal{C}}(M X, M X)$, via the homotopy equivalences (25). We obtain a homotopy commutative diagram like (26), above, and conclude that $\eta_{a}:(M X)(a) \longrightarrow(M X)(a)$ is a weak homotooy equivalence for each object $a$ of $\mathcal{C}$. Hence $\eta: M X \longrightarrow M X$ is a weak equivalence of $\mathcal{C}$-diagrams. Since $M X$ is cofibrant-fibrant, by Whitehead's Theorem $\eta_{a}$ is a homotopy equivalence. But $M X$ is also a minimal $\mathcal{C}$-diagram, hence $\eta$ is indeed an isomorphism by Corollary 3.12. Thus $\eta$ is really a vertex of aut $\mathcal{C}(M X)$.

Proof of Theorem $\square$. We are assuming that $\mathcal{C}$ is a small artinian EI-category, $B$ a connected simplicial set, and $F$ an arbitrary $\mathcal{C}$-diagram. Let $M F$ be a minimal cofibrant-fibrant replacement for $F$.

Since $M F$ is minimal, a $\mathcal{C}$-fibre bundle over $B$ with fibre $M F, \xi: E \longrightarrow B$, is indeed a minimal fibration. For each object $c$ of $\mathcal{C},(M F)(c)$ is a Kan complex and $\xi(c): E(c) \longrightarrow B$ is a fibre bundle with fibre $(M F)(c)$, hence a fibration (cf. [1]). Thus, $\xi: E \longrightarrow B$ is a fibration of $\mathcal{C}$-diagrams. 
That $\xi$ is minimal follows from Proposition 3.9 . If $\left.\xi\right|_{D}: D \longrightarrow B$ is a strong fibrewise deformation retract of $\xi$, then for each simplex $\sigma$ of $B$, the pullback along the inclusion of the simplex $\sigma: \Delta[n] \longrightarrow B$ produces a retraction

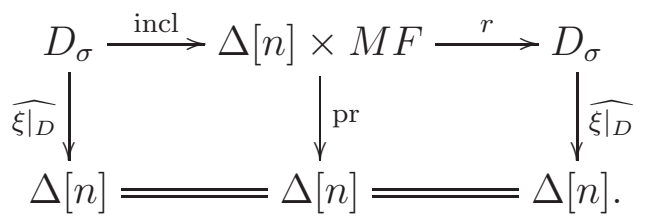

Moreover, since $M F$ is minimal, pr: $\Delta[n] \times M F \longrightarrow \Delta[n]$ is a minimal fibration by Lemma 5.1. Then, Proposition 3.9 implies that incl: $D_{\sigma} \longrightarrow \Delta[n] \times M F$ is an isomorphism, and therefore incl $: D \longrightarrow E$ is an isomorphism. Now, Proposition 3.9 implies that $\xi: E \longrightarrow B$ is minimal.

Now we can define the correspondence

$$
\left\{\begin{array}{l}
\text { Equivalence classes of } \\
\mathcal{C} \text {-fibre bundles over } \\
B \text { with fibre } M F
\end{array}\right\} \stackrel{J}{\longrightarrow}\left\{\begin{array}{l}
\text { Weak homotopy classes of } \\
\text { fibrations of } \mathcal{C} \text {-diagrams over the } \\
\text { constant diagram } B \text { and fibre } \\
\text { weakly homotopy equivalent to } F
\end{array}\right\}
$$

by assigning to the class of $\mathcal{C}$-fibre bundle $\xi: E \longrightarrow B$ the weak homotopy class of the same map as a fibration of $\mathcal{C}$-diagrams with fibre $M F \simeq_{w} F$.

Proposition 5.9(b) together with Corollary 5.7 imply that $J$ is surjective while Proposition 5.10(b) implies that it is injective. Then, the result follows from Theorem B and Proposition 5.14.

\section{Appendix A. Preordered sets}

Let $A$ be a set and ' $\preceq$ ' a preorder relation over $A$ (i.e., $\preceq$ is reflexive and transitive). For this set we need to find a subset $A^{\prime}$ of $A$ satisfying the conditions:

R1: For all $w \in A$ there exists $x \in A^{\prime}$ with $x \preceq w$.

R2: $A^{\prime}$ is minimal among subsets satisfying $\mathrm{R} 1$.

There is a simple example which shows that $A^{\prime}$ does not always exist: consider an infinite sequence of sets and maps

$$
\ldots \longrightarrow A_{n} \stackrel{f_{n}}{\longrightarrow} \ldots \longrightarrow A_{1} \stackrel{f_{1}}{\longrightarrow} A_{0}
$$

with nonempty inverse limit. Let us define the relation ' $\preceq$ ' over the disjoint union $A=$ $\coprod_{i \geq 0} A_{i}$ as follows: given $a \in A_{j}$ and $a^{\prime} \in A_{k}$ we say that $a \preceq a^{\prime}$ if there exists a map $f: A_{j} \longrightarrow A_{k}$ such that $f(a)=a^{\prime}$. In this case there is no subset $A^{\prime} \subseteq A$ satisfying both conditions R1 and R2. In fact, any element in the inverse limit provides an infinite sequence $a_{0} \succeq a_{1} \succeq \cdots \succeq a_{i} \succeq \ldots$, in $A$, with $a_{i} \in A_{i}$, which does not stabilize.

We can reformulate this problem by defining an order relation over a convenient quotient of $A$, that is, over the preordered set $(A, \preceq)$ we define the following relation: given $x, w \in A$ we say that $x \sim w$ if $x \preceq w$ and $w \preceq x$. Note that ' $\sim$ ' is an equivalence relation over $A$. Now, we define an partial order relation over $A / \sim$. Given classes $[x],[w] \in A / \sim$ we set

$$
[x] \leq[w] \quad \text { if } \quad x \preceq w .
$$

With this new relation the couple $(A / \sim, \leq)$ is a partially ordered set, and then the existence of a subset $A^{\prime}$ satisfying the above required conditions depends on the existence of minimal elements in every maximal chain of $A / \sim$. 
Let $\mathcal{C}$ be a small category and let $X: \mathcal{C} \longrightarrow$ Sets be a functor. We will say that $X$ is a diagram of sets and equivalence relations if for every object $c$ of $\mathcal{C}$ there is an equivalence relation $\sim_{c}$ defined over $X_{c}$, and is natural in the sense that for every $f: c \longrightarrow d$ in $\mathcal{C}$, if $x \sim_{c} x^{\prime}$ then $X_{f}(x) \sim_{d} X_{f}\left(x^{\prime}\right)$.

Under these circumstances we can define a new induced relation $\preceq$ over the disjoint union $A=\coprod_{c \in \mathrm{Ob}(\mathcal{C})} X_{c}$ as follows: given $x \in X_{c}$ and $w \in X_{d}$ we say that $x \preceq w$ if there exists a morphism $f: c \longrightarrow d$ in $\mathcal{C}$ such that $X_{f}(x) \sim_{d} w$. Now, the pair $(A, \preceq)$ is a preordered set.

Proposition A.1. Let $\mathcal{C}$ be an artinian EI-category. If $X: \mathcal{C} \longrightarrow$ Sets is a diagram of sets and equivalence relations, then the set $A=\coprod_{c \in \mathrm{Ob}(\mathcal{C})} X_{c}$ equipped with the induced preorder relation $\preceq$ defined above contains a subset $A^{\prime}$ satisfying the conditions $R 1$ and $R 2$.

Proof. Define an equivalence relation $\sim$ on $A$ by setting $x \sim x^{\prime}$ if both $x \preceq x^{\prime}$ and $x^{\prime} \preceq x$. Write $[x]$ for the class of $x$ in $A / \sim$. Now, define a partial order relation on $A / \sim$ by setting $[x] \leq[y]$ if $x \preceq y$. Consider the set of all maximal chains of $A / \sim$ :

$$
\mathcal{F}=\{C \subseteq A / \sim \mid C \text { is a maximal chain }\}
$$

Take an arbitrary maximal chain $C=\left\{\left[x_{i}\right]\right\}_{i \in I}$ in $A / \sim$ with $x_{i} \in X_{c_{i}}, c_{i} \in \mathrm{Ob}(\mathcal{C})$, for each $i \in I$. The classes $\left[c_{i}\right]$ of these objects $c_{i}, i \in I$, form a chain in $\mathcal{C} / \sim$, so it stabilizes since $\mathcal{C}$ is artinian (see Definition [3.5). In fact, given two of these objects $c_{i}$ and $c_{j}$, we have elements $x_{i} \in X_{c_{i}}$ and $x_{j} \in X_{c_{j}}$ with $\left[x_{i}\right],\left[x_{j}\right] \in C$, thus either $\left[x_{i}\right] \leq\left[x_{j}\right]$ in which case there is a morphism $f: c_{i} \longrightarrow c_{j}$ in $\mathcal{C}$ and $\left[c_{i}\right] \leq\left[c_{j}\right]$, or $\left[x_{j}\right] \leq\left[x_{i}\right]$ in which case $\left[c_{j}\right] \leq\left[c_{i}\right]$.

There is a representative $c_{0} \in \mathrm{Ob} \mathcal{C}$ of the minimal element $\left[c_{0}\right]$ in the chain of classes of objects such that there is an element $x_{0} \in X_{c_{0}}$ with $\left[x_{0}\right] \in C$. It turns out that $\left[x_{0}\right]$ is minimal in $C$. Assume $[z] \in C$ and $[z] \leq\left[x_{0}\right]$, then $z \in X_{d}$ for some object $d$ of $\mathcal{C}$ and there is a morphism $f: d \longrightarrow c_{0}$ in $\mathcal{C}$ such that $X(f)(z) \simeq_{c_{0}} x_{0}$. Then, $[d]$ belongs to the chain of objects and $[d] \leq\left[c_{0}\right]$, hence $[d]=\left[c_{0}\right]$ since $\left[c_{0}\right]$ is minimal. That is, we also have $\left[c_{0}\right] \leq[d]$, so there is a morphism $g: c_{0} \longrightarrow d$. Since $\mathcal{C}$ is an $E I$-category, the composition $\varphi=g \circ f$ is an automorphism of $d$. Upon replacing $g$ with $\varphi^{-1} \circ g$ we can assume that $g \circ f=\operatorname{Id}_{d}$. It follows that $z=X(g)(X(f)(z)) \simeq_{d} X(g)\left(x_{0}\right)$, so $x_{0} \preceq z$ and $\left[x_{0}\right] \leq[z]$. Hence $[z]=\left[x_{0}\right]$.

Consider now the set $M$ of minimal elements of the chains in $\mathcal{F}$, that is

$$
M=\{[x] \in A / \sim \mid \text { there is } C \in \mathcal{F} \text { such that }[x] \text { in minimal in } C\}
$$

By the Axiom of Choice, there is a subset $A^{\prime}$ that contains one and only one representative in each of the classes that form the set $M$.

We will show that $M$ satisfies conditions $\mathrm{R} 1$ and R2. Fix $w \in A$, so that $[w]$ belongs to a maximal chain $C \subseteq A / \sim$. Then, there is $x \in A^{\prime}$ such that $[x]$ is minimal in $C$. It follows that $[x] \leq[w]$, thus $x \preceq w$. This proves R1.

Assume that there is another subset $B, B \subseteq A^{\prime} \subseteq A$ satisfying R1. Fix an element $w \in A^{\prime}$. There must be an element $x \in B, x \preceq w$, since $B$ satisfies R1. Hence $[x] \leq[w]$. By definition of $A^{\prime}$, there is a maximal chain $C$ in $A / \sim$ such that $[w]$ is minimal in $C$. Then $D=\{[x]\} \cup C$ is also a chain and it contains $C$, hence it must be $C$, so $[x] \in C$. Therefore $[x]=[w]$, but $x \in B \subset A^{\prime}$, so both $x$ and $w$ are in $A^{\prime}$, and actually $x=w$. This proves that $A^{\prime} \subset B$ and hence that $A^{\prime}$ satisfies R2.

Remark A.2. Notice that the choice of the subset $A^{\prime}$ in Proposition A.1 could be refined by choosing elements satisfying further properties. Let $P: A \rightarrow\{0,1\}$ be a function defined 
on $A$ and, using the same notation as in the proof of the proposition, set

$$
\widehat{[x]}= \begin{cases}{[x],} & \text { if } P(y)=1 \text { for all } y \in[x], \\ \{y \in[x] \mid P(y)=0\}, & \text { otherwise. }\end{cases}
$$

for each class $[x]$ of $A / \sim$ which is minimal in a chain in $(A / \sim, \leq)$. Then, by choosing an

element in each of the sets $\widehat{[x]}$ we obtain a refined subset $A^{\prime}$ that satisfies R1, R2, and for each $x \in A^{\prime}, P(x)=0$ if and only if there is $y \in[x]$ with $P(y)=0$.

\section{REFERENCES}

[1] M.G. Barratt, V.K. Gugenheim and J.C. Moore, On Semisimplicial fibre bundles, Amer. J. Math. 81 (1959), 639-657.

[2] M. Blomgren and W. Chachólski, On the classification of fibrations, Trans. Amer. Math. Soc. 367 (2015), 519-557.

[3] A.K. Bousfield, Homotopical localizations of spaces, Amer. J. Math. 119 (1997), no. 6, 1321-1354.

[4] A.K. Bousfield and D. Kan, Homotopy limits, completions and localizations, Lecture Notes in Math., 304, Springer (1972)

[5] E. Curtis, Simplicial Homotopy Theory, 6, Adv. Math. (1971).

[6] W. Dwyer and D. Kan, Function complexes for diagrams of simplicial sets, Nederl. Akad. Wetensch. Indag. Math. 86, (1983), 139-147.

[7] W. Dwyer, D. Kan and J. Smith, Towers of fibrations and homotopical wreath products, J. Pure Appl. Alg. 56 (1989), 9-28.

[8] E. Farjoun and J. Smith, Homotopy localization nearly preserves fibrations, Topology 34 (1995), no. $2,359-375$.

[9] R. Fritsch and R. Piccinini, Cellular structures in Topology, 19, Cambridge University Press (1990).

[10] P. Goerss, J. Jardine, Simplicial Homotopy Theory, Birkhäuser Verlag (1999).

[11] V. Gugenheim, On supercomplexes, Trans. Amer. Math. Soc. 85 (1957), 35-51.

[12] P. Hirschhorn, Model categories and their localization, Mathematical Surveys and Monographs 99, AMS (2003).

[13] M. Hovey, Model categories, Mathematical Surveys and Monographs 63, AMS (1999).

[14] W. Lück, Transformation groups and algebraic K-theory, Lecture Notes in Math., 1408, SpringerVerlag (1980).

[15] J.P. May, Simplicial objects in Algebraic Topology, Van Nostrand, Princeton (1967).

[16] D. Quillen, Homotopical Algebra, Lecture Notes in Math., 43. Springer-Verlag, Berlin-New York (1967).

Departament de Matemàtiques, Universitat Autònoma de Barcelona, E-08193 Bellaterra, SPAIN

E-mail address: broto@mat.uab.cat

Departamento de Geometría y Topología, Universidad de Sevilla, E-41012, Sevilla, Spain E-mail address: ramonjflores@us.es

Facultad de Ciencias Naturales y Matemáticas, Universidad del Rosario, 111711 Bogotá, Colombia

E-mail address: carlosan.giraldo@urosario.edu.co 